\title{
Census of Quadrangle Groups Inclusions
}

\author{
António Breda d'Azevedo, Domenico A. Catalano, Ján Karabáš \\ and Roman Nedela
}

\begin{abstract}
In a classical result of 1972 Singerman classifies the inclusions between triangle groups. We extend the classification to a broader family of triangle and quadrangle groups forming a particular subfamily of Fuchsian groups. With two exceptions, each inclusion determines a finite bipartite map (hypermap) on a 2dimensional spherical orbifold that encodes the complete information and gives a graphical visualisation of the inclusion. A complete description of all the inclusions is contained in the attached tables.
\end{abstract}

\section{Introduction}

The search for inclusions between triangle groups, and more generally between Fuchsian groups, was motivated by the theory of Riemann surfaces and algebraic geometry. Triangle and quadrangle groups are particular instances of Fuchsian groups, which are finitely generated discrete subgroups of $\operatorname{PSL}(2, \mathbb{R})$, the group of conformal automorphisms of the upper-half plane. Inclusions of Fuchsian groups played an important rôle in the investigation of Teichmüller spaces, see for instance Greenberg [10, Theorem 1]. Later Singerman extended some of Greenberg's results and obtained a complete list of normal inclusions between Fuchsian groups having the

\author{
A.B. d'Azevedo · D.A. Catalano \\ Departamento de Matemática, Universidade de Aveiro, 3810-193 Aveiro, Portugal \\ e-mail: breda@ua.pt \\ D. A. Catalano \\ e-mail: domenico@ua.pt \\ J. Karabáš $(\bowtie)$ \\ Department of Computer Science, Faculty of Natural Sciences, Matej Bel University, \\ 97401 Banská Bystrica, Slovakia \\ e-mail: karabas@savbb.sk \\ R. Nedela \\ University of West Bohemia, Pilsen, Czech Republic \\ e-mail: nedela@ntis.zcu.cz


same Teichmüller space dimension. In addition, he gives all non-normal inclusions between triangle groups [14, 15]. Another motivation for looking at inclusions of Fuchsian groups in triangle groups comes from the connection between algebraic curves over complex numbers, Riemann surfaces and dessins d'enfant, established explicitly by a result of Belyı̆ [1], see also [17]. It follows that every hypermap endows its underlying closed orientable surface with a complex structure by lifting the complex structure of the Riemann sphere via a Bely $\breve{1}$ function, a meromorphic function ramified above at most three points (located at 0,1 and $\infty$ ). A natural question arises: Under which conditions do two hypermaps determine the same Riemann surface? In certain circumstances, inclusions of Fuchsian groups in triangle groups with spherical quotients correspond to Riemann-surface-preserving transformations of hypermaps, see $[5,16]$.

The main aim of this paper is to present a complete list of finite index inclusions $P<Q$, with both $P$ and $Q$ being either a triangle or a quadrangle group (with finite periods). In what follows, we give an outline of the proof followed by instructions how to read the attached census.

\section{Generalised Quadrangle Groups and Constellations}

Quadrangle groups. By a generalised quadrangle group we mean a Fuchsian group $Q$ with presentation

$$
Q(k, l, m, n)=\left\langle x, y, z, w \mid x^{k}=y^{l}=z^{m}=w^{n}=x y z w=1\right\rangle,
$$

where $k, l, m, n$ are positive integers satisfying $\frac{1}{k}+\frac{1}{l}+\frac{1}{m}+\frac{1}{n}<2$. Clearly, at most one of $k, l, m, n$ can be equal to one. Therefore a generalised quadrangle group is either a triangle or a quadrangle group. In what follows, we assume that the parameters $k, l, m$, and $n$ are ordered in a non-decreasing order. This is motivated by the fact that a permutation of the parameters (or of the generators) in the above presentation gives an isomorphic copy of $Q(k, l, m, n)$. In particular, the group $Q(1, l, m, n)$ is just the triangle group $\Delta(l, m, n)$. Inclusions between triangle groups were classified by Singerman in [15] and they are listed in Appendix (see Tables 1 and 2).

Constellations. Let $P=Q(p, q, r, s)$ and $Q=Q(k, l, m, n)$ be two generalised quadrangle groups and let $P$ be a subgroup of index $N$ in $Q$. We write $P<_{N} Q$. The meaning of the parameters $N, p, q, r, s, k, l, m$, and $n$ will be fixed throughout the whole paper. There is an induced action of $Q$ on the (right) cosets of $P$ represented by four permutations $\pi_{x}, \pi_{y}, \pi_{z}, \pi_{w}$ corresponding to the images of the four generators of $Q$ in the natural homomorphism into the symmetric group $\operatorname{Sym}(N)$. In accordance with Lando and Zvonkin [13, Chap. 1], we call the four-tuple $\mathscr{C}=\left[\pi_{x}, \pi_{y}, \pi_{z}, \pi_{w}\right]$ a constellation (or a 4-constellation) of degree $N$ and the sequence $\left[\lambda_{x}, \lambda_{y}, \lambda_{z}, \lambda_{w}\right]$ of partitions of $N$, where each $\lambda_{a}$ is the cycle structure of the permutation $\pi_{a}$, the passport of the constellation $\mathscr{C}$. The monodromy group $\operatorname{Mon}(\mathscr{C})$ of the constellation $\mathscr{C}$ is the group $\left\langle\pi_{x}, \pi_{y}, \pi_{z}, \pi_{w}\right\rangle \leq \operatorname{Sym}(N)$. By definition, the action of $\operatorname{Mon}(\mathscr{C})$ is transitive on the set $\{1,2, \ldots, N\}$ and $\pi_{x} \pi_{y} \pi_{z} \pi_{w}=1$. We write the cycle structure 
of a permutation in the exponential notation: for instance, the permutation $\varrho=$ $(1,2,3)(4,5)(6,7,8)(9)$ has the cycle structure [1.2.3 $\left.{ }^{2}\right]$. For convenience, each cycle structure in a passport in the census is ordered in a non-decreasing order.

Two constellations $\mathscr{C}=\left[\pi_{x}, \pi_{y}, \pi_{z}, \pi_{w}\right]$ and $\mathscr{C}^{\prime}=\left[\pi_{x}^{\prime}, \pi_{y}^{\prime}, \pi_{z}^{\prime}, \pi_{w}^{\prime}\right]$ of degree $N$ are equivalent if there exists $\alpha \in \operatorname{Sym}(N)$ such that the corresponding permutations are simultaneously conjugated by $\alpha$. In particular, if $P<Q$ and $P^{\prime}<Q$ are two inclusions of generalised quadrangle groups, then the associated constellations are equivalent if and only if the subgroups $P$ and $P^{\prime}$ are conjugate in $Q$. In fact, an inclusion $P<Q$ determines a constellation $\mathscr{C}=\left[\pi_{x}, \pi_{y}, \pi_{z}, \pi_{w}\right]$ which corresponds to a $Q$-marked hypermap $\mathscr{P}=\left(Q / P ; x P^{*}, y P^{*}, z P^{*}\right)$, where $P^{*}$ is the core of $P$ in $Q$ and $\pi_{x}, \pi_{y}, \pi_{z}$ are the actions of $x P^{*}, y P^{*}, z P^{*}$ on the $N$ cosets of $P$ in $Q$ respectively (see [3] for definitions). Replacing $\Theta$ by $Q$ in [3, Theorem 19], we have that two $Q$-marked hypermaps $\mathscr{P}$ and $\mathscr{K}=\left(Q / K ; x K^{*}, y K^{*}, z K^{*}\right)$, corresponding to the inclusions $P<Q$ and $K<Q$ respectively, are isomorphic if and only if $P$ and $K$ are conjugate in $Q$, say $K=P^{g}$, for some $g \in Q$; the isomorphism is the conjugation morphism $\iota^{g}: Q / P \rightarrow Q / K, P q \mapsto Q^{g} q^{g}$. If $\psi_{1}: Q / P \rightarrow\{1,2, \ldots, N\}$ and $\psi_{2}: Q / K \rightarrow\{1,2, \ldots, N\}$ are the bijections to their transversals, then $\alpha=\psi_{1}^{-1} \iota^{g} \psi_{2}$ is the permutation that makes the constellations corresponding to $\mathscr{P}$ and $\mathscr{K}$ equivalent.

Our approach will follow the one outlined in Singerman's classification of inclusions between triangle groups [15]. The census is obtained in two steps. First, we find all admissible sets of parameters $N ; p, q, r, s ; k, l, m, n$ satisfying the RiemannHurwitz equation with additional numerical constrains. Each such numerical solution gives rise to a passport. Secondly, for each passport we either find all equivalency classes of constellations with that passport, or we show that such a constellation does not exist. Both steps are computer-assisted. As a byproduct, we confirm Singerman's classification of triangle group inclusions. More details follow.

Numerical solution. If we have an inclusion between generalised quadrangle groups $P<_{N} Q$ with parameters $N ; p, q, r, s ; k, l, m, n$, then the Riemann-Hurwitz formula holds true

$$
N=\frac{2-\left(\frac{1}{p}+\frac{1}{q}+\frac{1}{r}+\frac{1}{s}\right)}{2-\left(\frac{1}{k}+\frac{1}{l}+\frac{1}{m}+\frac{1}{n}\right)}
$$

Our aim is to determine all possible solutions $N ; p, q, r, s ; k, l, m, n$ with their associated passports. To do this, the following two well known facts are useful:

- elements of finite order in $P S L(2, \mathbb{R})$ are elliptic,

- any element $g$ of finite order in a Fuchsian group is conjugate to a power of an elliptic generator $h$; in symbols $g \vdash h$.

Let $a, b, c, d$ be the generators of $P$ of orders $p, q, r, s$, respectively. If $P<Q$, then one of the following four cases (up to a group isomorphism) happens:

Case 1. $a, b, c, d \vdash w$, which implies $p|n, q| n, r \mid n$ and $s \mid n$,

Case 2. $a \vdash z$ and $b, c, d \vdash w$, which implies $p \mid m$ and $q|n, r| n$ and $s \mid n$, 
Case 3. $a, b \vdash z$, and $c, d \vdash w$, which implies $p|m, q| m$ and $r|n, s| n$

Case 4. $a \vdash y, b \vdash z$ and $c, d \vdash w$, which implies $p|l, q| m$ and $r|n, s| n$.

Remark It might appear that the case $a \vdash x, b \vdash y, c \vdash z$ and $d \vdash w$ is missing, however (1) implies $N=1$, that is, $P=Q$ in this case.

Using an argument by Singerman [14, Theorem 1], if there is a constellation associated to one of the aforementioned cases, then it has one of the following passports, according to the case it belongs to:

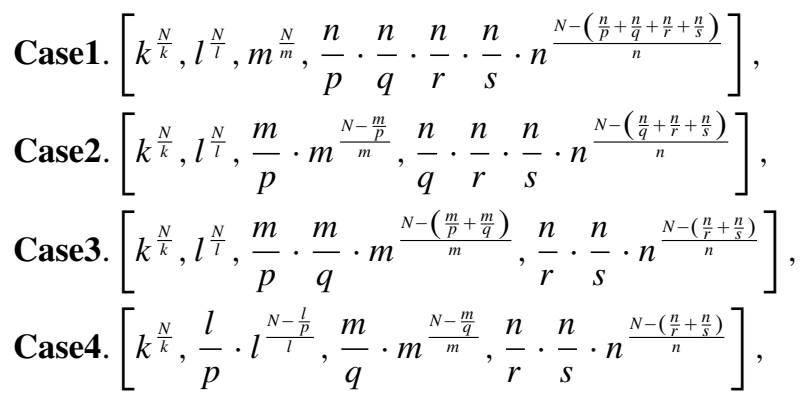

where all of the fractions appearing in the above passports are integers. We adopt the convention that factors with zero exponent in passports are vacuous (and are not to be taken as equal to 1 ). For instance, $\frac{n}{r} \cdot \frac{n}{s} \cdot n^{0}$ should be interpreted as $\frac{n}{r} \cdot \frac{n}{s}$ and not as $\frac{n}{r} \cdot \frac{n}{s} \cdot 1$, which has a different meaning in a passport.

Each passport which belongs to one of the above four cases with parameters satisfying (1) will be called admissible. Admissible passports may or may not be passports of constellations.

By definition, the length of each cycle of $\pi_{x}$ (resp. $\pi_{y}, \pi_{z}$ and $\pi_{w}$ ) in a constellation is a divisor of $k\left(l, m\right.$, and $n$, respectively). A cycle of a permutation $\pi_{x}, \pi_{y}, \pi_{z}$ or $\pi_{w}$ will be called singular if its length is strictly less then the order $k, l, m$ or $n$ of the corresponding generator $x, y, z$ or $w$. If $p \neq 1$, that is, if $P$ is not a triangle group, then each admissible passport has exactly four cycle lengths that are proper divisors of $k, l, m$ or $n$. If $P$ is a triangle group $(p=1)$, then by [15, Proposition 5 and Theorem 1], $Q$ is a triangle group as well, or equivalently $k=1$. In this case there are exactly three singular cycles in the constellation associated with the inclusion $P<Q$. The four types of passports are distinguished by their respective distributions of the singular cycles between the four permutations.

As we already mentioned, not every admissible passport can be realised by an inclusion $P<_{N} Q$. To determine the inclusions that realise admissible passports, we used computer algebra systems MAGMA [2] and GAP [9]. With the exception of two families described in Table 3, each inclusion $P<_{N} Q$ has the parameter $k$ equal to 1 , which means that $Q$ is a triangle group. In this case $Q$ can be viewed as a group of orientation preserving automorphisms of an infinite regular hypermap (or bipartite map) $\mathbf{U}$ on the hyperbolic plane with hypervertices (black vertices) of valency $l$, hyperedges (white vertices) of valency $m$ and hyperfaces of valency $n$ (faces of valency $2 n$ ). Then $P$ is a group of automorphisms of $\mathbf{U}$ and the quotient 
$\mathbf{U} / P$ is a hypermap on the Riemann sphere ( $P$ is a Fuchsian group of signature $(0 ;\{p, q, r, s\}))$, more precisely, the quotient hypermap lies on a spherical orbifold with exactly 4 or 3 singular points corresponding to the singular cycles of the constellation associated with the inclusion $P<Q$.

Dessins d'enfant. When $Q$ is a triangle group, $k=1$ and therefore $\pi_{x}=1$. Then the inclusion $P<_{N} Q$ gives rise to a 4-constellation $\mathscr{C}$ that can be reduced to the 3 -constellation $\left[\pi_{y}, \pi_{z}, \pi_{w}\right]$. This can be regarded as a spherical hypermap $\mathscr{H}$ on the set $\{1,2, \ldots, N\}$, whose hypervertices and hyperedges are the orbits of $\pi_{y}$ and $\pi_{z}$, respectively. The hyperfaces of $\mathscr{H}$ are the orbits of $\pi_{w}$.

There exists a regular branched covering from the universal infinite hypermap $\mathbf{U}=\mathbf{U}(l, m, n)$ on the hyperbolic plane onto the spherical hypermap $\mathbf{U} / P$ either with exactly four branched points with indexes $p, q, r, s$, or with exactly three branched points with indexes $q, r, s$, located at some hypervertices, hyperedges or hyperfaces. This hypermap (on the spherical orbifold) with the additional information about the branched points and their respective indices will be called a dessin d'enfant or simply a dessin. It is more precise to talk about a hypermap on an orbifold with signature $(0 ;\{p, q, r, s\})$, or with signature $(0 ;\{q, r, s\})$ when $p=1$, rather than simply talking of a "hypermap". There is a one-to-one correspondence between the singular cycles of $\pi_{y}, \pi_{z}$, and $\pi_{w}$ and the branched points of the associated dessin.

Recall that the monodromy group $\operatorname{Mon}(\mathscr{C})$ of the constellation associated to an inclusion $P<_{N} Q$ acts transitively on $\{1,2, \ldots, N\}$. Moreover, Mon( $\left.\mathscr{C}\right)$ acts regularly on $\{1,2, \ldots, N\}$ if and only if $P \triangleleft_{N} Q$ is a normal inclusion. In this case the associated dessin is also called regular.

Families of inclusions. The inclusions may form infinite families parametrised by one, two or three integer parameters in the signatures (corresponding to the number of zero exponents in the factors of the respective passport). All the inclusions of an infinite family share the same (non-parametrised) passport. Note that each member of an infinite family is represented by the same hypermap in the census. An inclusion not belonging to an infinite family is called sporadic.

Although there are infinitely many inclusions, the number of admissible passports is finite. Each admissible passport gives rise to a finite number of constellations (or dessins), since the index $N$ of any inclusion is finite and bounded by 84 (the RiemannHurwitz bound). Thus, the identification of all dessins associated to quadrangle group inclusions is a finite problem.

More details (and proofs) on the classification of inclusions of generalised quadrangle groups will be discussed in the forthcoming paper [6].

\section{How to Read the Census}

The attached tables contain the complete list of inclusions $P<_{N} Q$ between generalised quadrangle groups. Two inclusions $P<_{N} Q$ and $P^{\prime}<_{N} Q$ are distinguished up to conjugation in $Q$; if $P^{\prime}=P^{g}$ for some $g \in Q$, then $P<_{N} Q$ and $P^{\prime}<_{N} Q$ give equivalent constellations and so the two inclusions are essentially the same. 
Each conjugacy class of inclusions $P<_{N} Q$ of generalised quadrangle groups forms one entry in the census. Excluding Table 3 (explained below), the corresponding row displays the following data:

- the associated passport $\lambda=\left[\lambda_{y}, \lambda_{z}, \lambda_{w}\right]$ (up to a permutation of its entries),

- the number of realisations, which is equal to the number of non-conjugate subgroups of $Q$ isomorphic to $P$,

- the non-isomorphic dessins with passport $\lambda$ up to mirror images,

- the monodromy group, or a structure description of the monodromy group, or the prime factor decomposition of its size $[8,9]$.

If two dessins form a chiral pair, only one member of the pair is depicted. Thus the number of the depicted dessins may not match the displayed number of realisations in the row.

The three cycle structures in a passport describe the following:

1. first item in a passport gives the sequence of hypervertex valencies (degrees of black vertices),

2. the second item gives the sequence of hyperedge valencies (degrees of white vertices), and

3. the third item gives the sequence of hyperface valencies.

However, there are 6 possible passports formed by permuting the entries of any given passport and therefore, each dessin $\mathscr{D}$ may be in principle associated with 5 (or 11, if $\mathscr{D}$ is chiral) additional non-isomorphic dessins. There is no essential reason to prefer any particular choice of one of these dessins for the census. The criteria we took into account were "aesthetic" — to indicate some symmetry of a dessin-or "space constraint" - a dual image of a dessin sometimes fits better into the reserved space - or we chose a dessin that was "triangulation resembling".

In Table 3 (when $Q$ is not a triangle group) the corresponding row displays:

- the associated passport $\lambda=\left[\lambda_{x}, \lambda_{y}, \lambda_{z}, \lambda_{w}\right]$ (up to a permutation of its entries),

- the associated constellation (since there is only one),

- an alternative picture based on $\Delta_{2}$-marked hypermaps [4]; these have blue, green and white vertices whose valencies (number of incidences of pairs of blue and green coloured edges $(b, g)$, in counter-clockwise order) give the first, the second and the third entry of the passport (the last entry corresponds to face-valencies),

- the monodromy group.

The entries of the census are organised into six tables. In each table, the inclusions (entries) are ordered by their indices in a non-decreasing order. The first two tables (Tables 1 and 2) include the case of normal and non-normal inclusions of triangle groups classified by Singerman [15]. Table 3 contains the two families of inclusions between (pure) quadrangle groups, while Table 4 gives a classification of normal inclusions of quadrangle groups in triangle groups. Table 5 lists the infinite families of non-normal inclusions of quadrangle groups in triangle groups. The longest table is Table 6, which contains the classification of the sporadic inclusions of quadrangle groups in triangle groups. 
A lot of information on inclusions can be dug from the tables. For instance:

- the indices of the inclusions cover all the integers from 2 to 22, additional integers covered are 24, 26, 27, 28, 29, 30, 36, 37, 44, 45, 52, 60;

- the largest possible index is 60 , realised by six inclusions of $Q(7,7,7,7)$ in $\Delta(2,3,7)$;

- the largest number of non-conjugate realisations (16) is achieved by the inclusion $Q(2,3,7,7)<\Delta(2,3,7)$ of index 44 ;

- the number of realisations of an inclusion varies from 1 to 16 . There are inclusions such that each of their realisations is chiral, such that each of their realisation is reflexible, and those that have both chiral and reflexible realisations.

For the sake of completeness, in Table 7 we collect all the solutions of (1) with the respective admissible passports for which there is no inclusion. Additional information on the inclusions of the generalised quadrangle groups can be found at the web page [7].

Acknowledgments This work was supported by the Research and Development Cooperation project FCT (Fundação para a Ciência e a Tecnologia) Portugal—Slovakia.

The work of the first two authors was partially supported by Portuguese funds through the CIDMA - Center for Research and Development in Mathematics and Applications (University of Aveiro), and the Portuguese Foundation for Science and Technology FCT, within project PEstOE/MAT/UI4106/2014.

The work of the third and the fourth author was supported by the Ministry of Education of the Slovak Republic, the grant VEGA 1/0150/14, by the project APVV SK-PT-0004-12, and by the project "Mobility-Enhancing Research, Science and Education," Matej Bel University (ITMS code 26110230082) under the Operational Programme of Education co-financed by the European Social Foundation.

The fourth author was supported by the project LO1506 of the Czech Ministry of Education, Youth and Sports.

The authors are very grateful to the anonymous referee for his/her careful and meticulous reading of the paper. The review was detailed and helpful to finalise the manuscript. 


\section{Appendix}

\section{Triangle Groups Inclusions}

See Tables 1 and 2.

Table 1 Normal inclusions of triangle groups

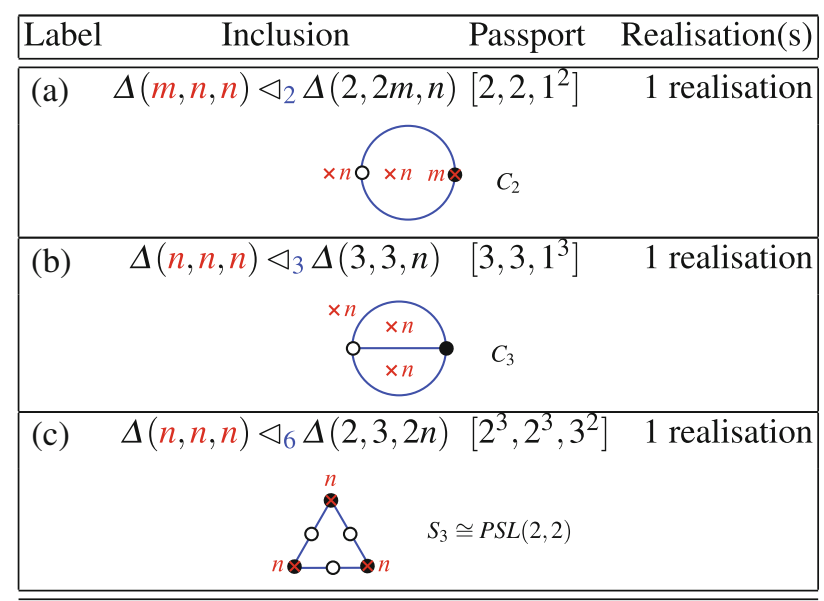


Table 2 Non-normal inclusions of triangle groups

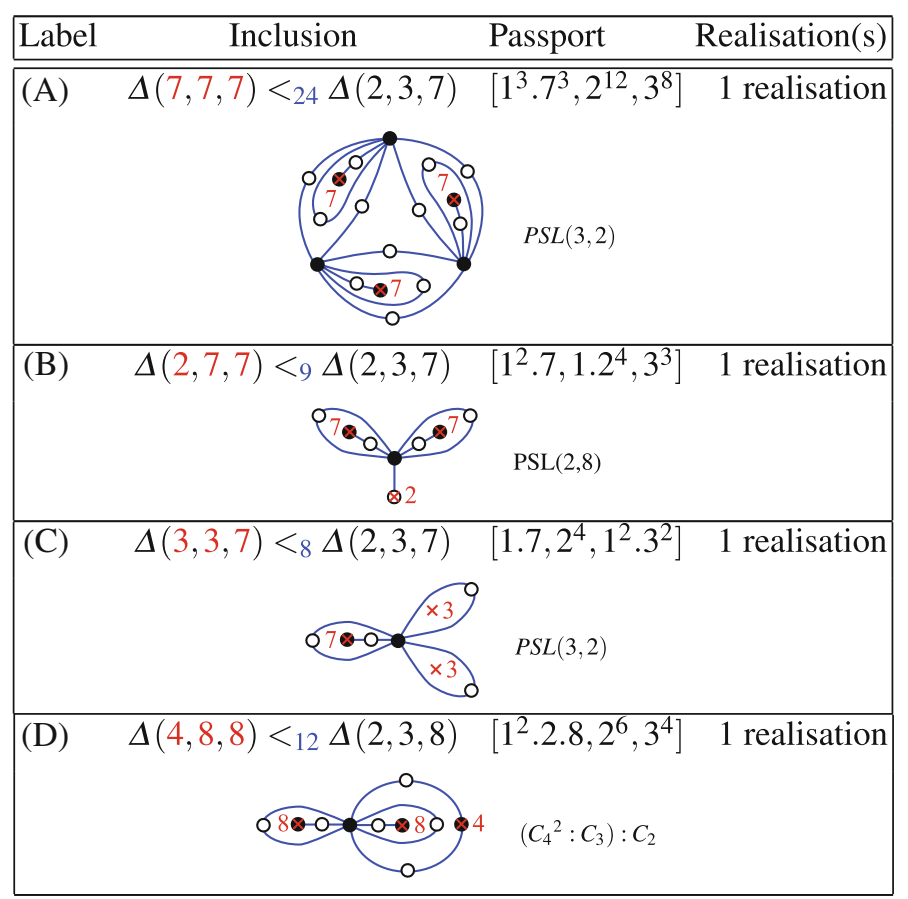

(continued) 
Table 2 (continued)

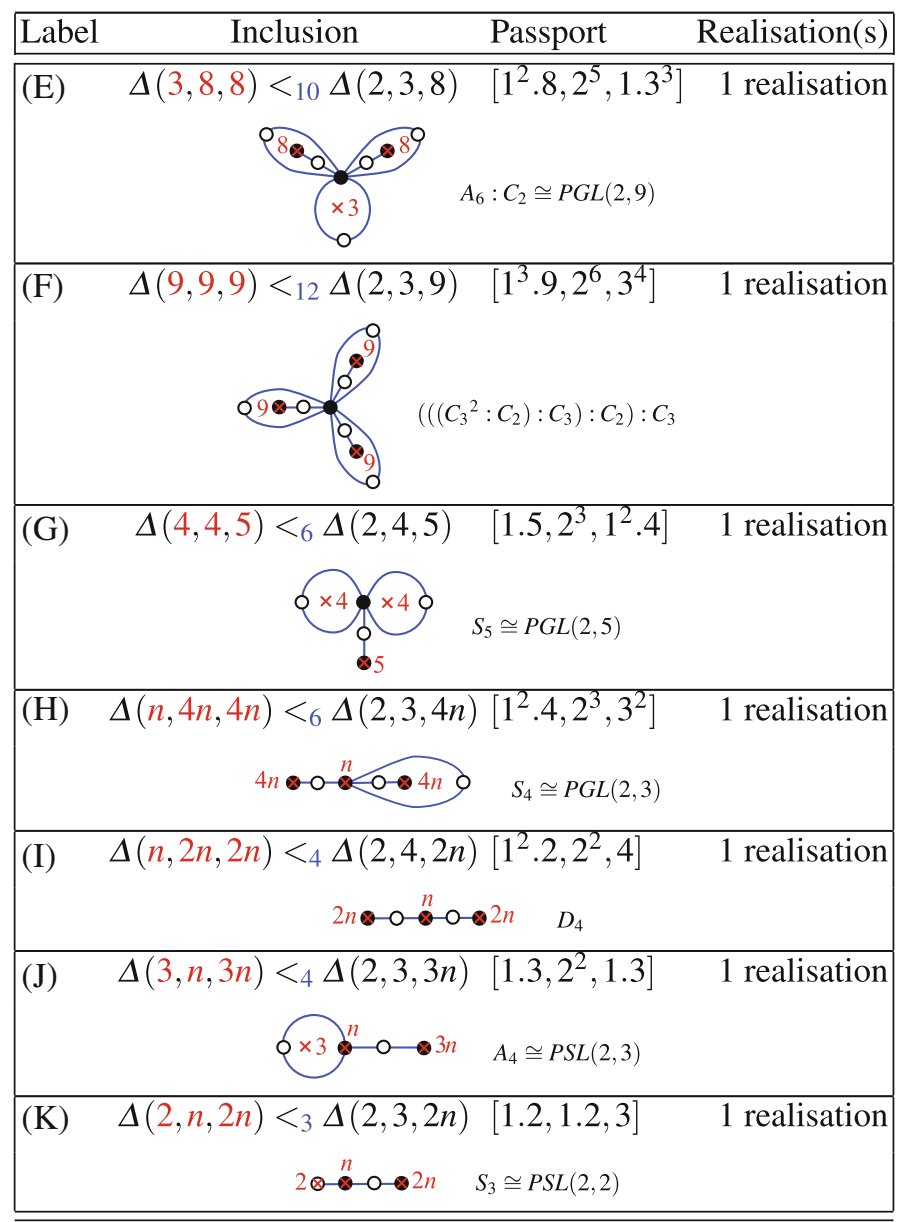




\section{Normal Inclusions of Quadrangle Groups}

See Tables 3 and 4.

Table 3 Inclusions of quadrangle groups in quadrangle groups

\begin{tabular}{|c|c|c|}
\hline Label & Inclusion & Constellation \\
\hline (Q1) & 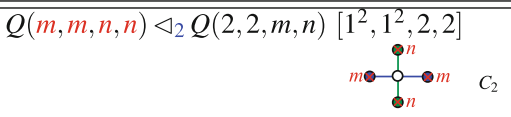 & {$\left[(),(),\left(\begin{array}{lll}1 & 2\end{array}\right),\left(\begin{array}{ll}1 & 2\end{array}\right)\right]$} \\
\hline (Q2) & $\left.Q(n, n, n, n) \triangleleft_{4} Q(2,2,2, n) 1^{4}, 2^{2}, 2^{2}, 2^{2}\right]$ & {$[(),(12)(34),(13)(24),(14)(23)]$} \\
\hline
\end{tabular}

Table 4 Infinite families of normal inclusions of quadrangle groups in triangle groups

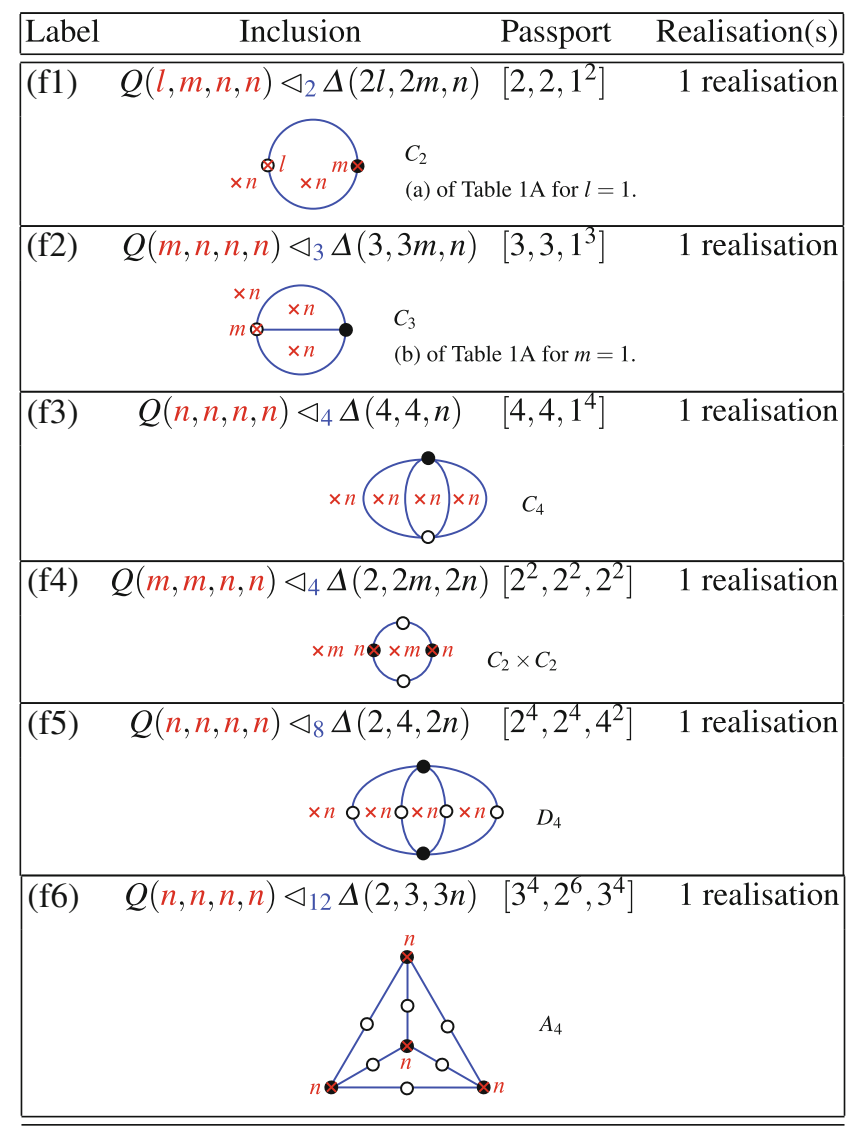




\section{Non-normal Inclusions of Quadrangle Groups}

See Tables 5 and 6.

Table 5 Infinite families of non-normal inclusions of quadrangle groups in triangle groups

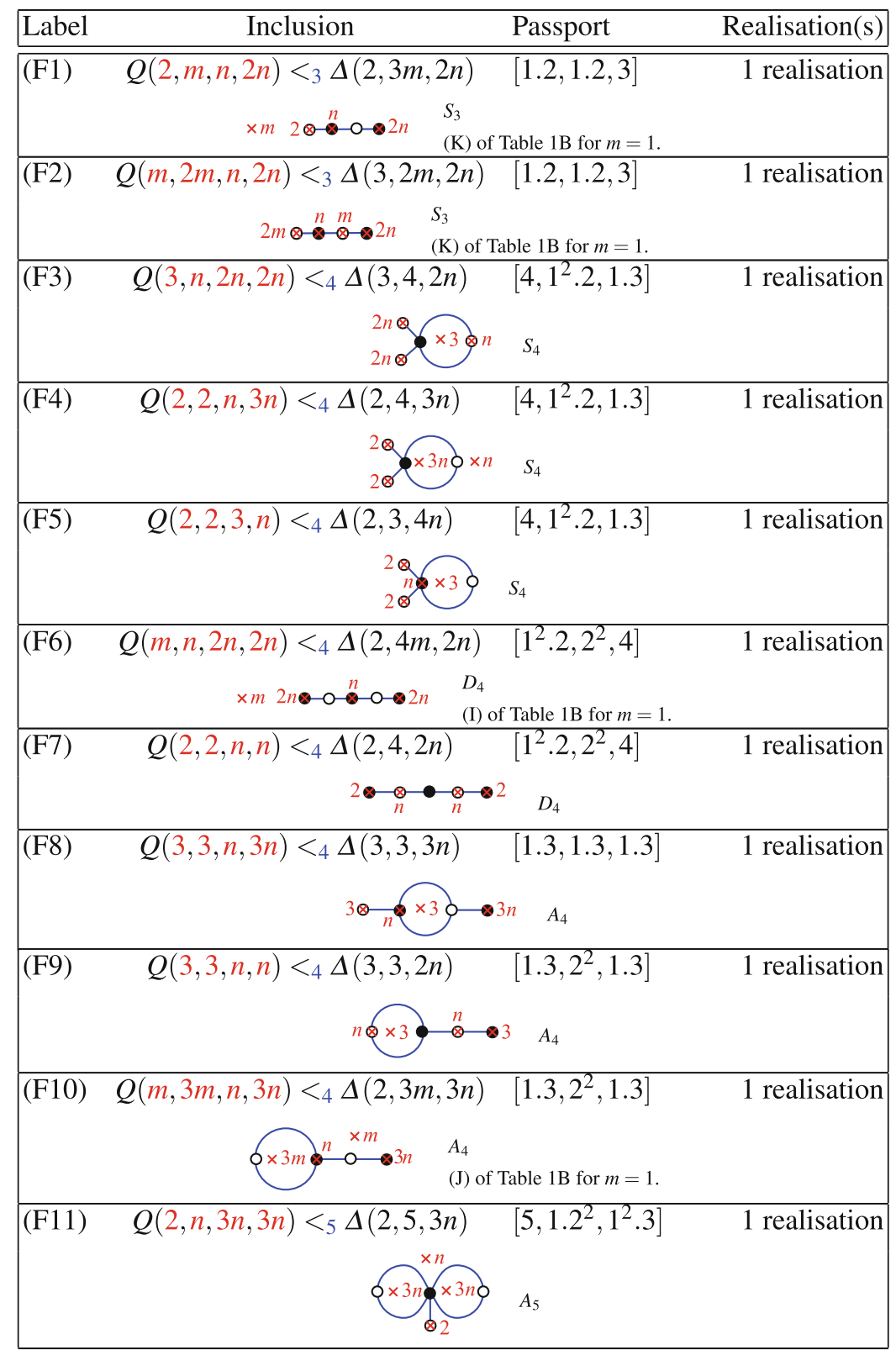


Table 5 (continued)

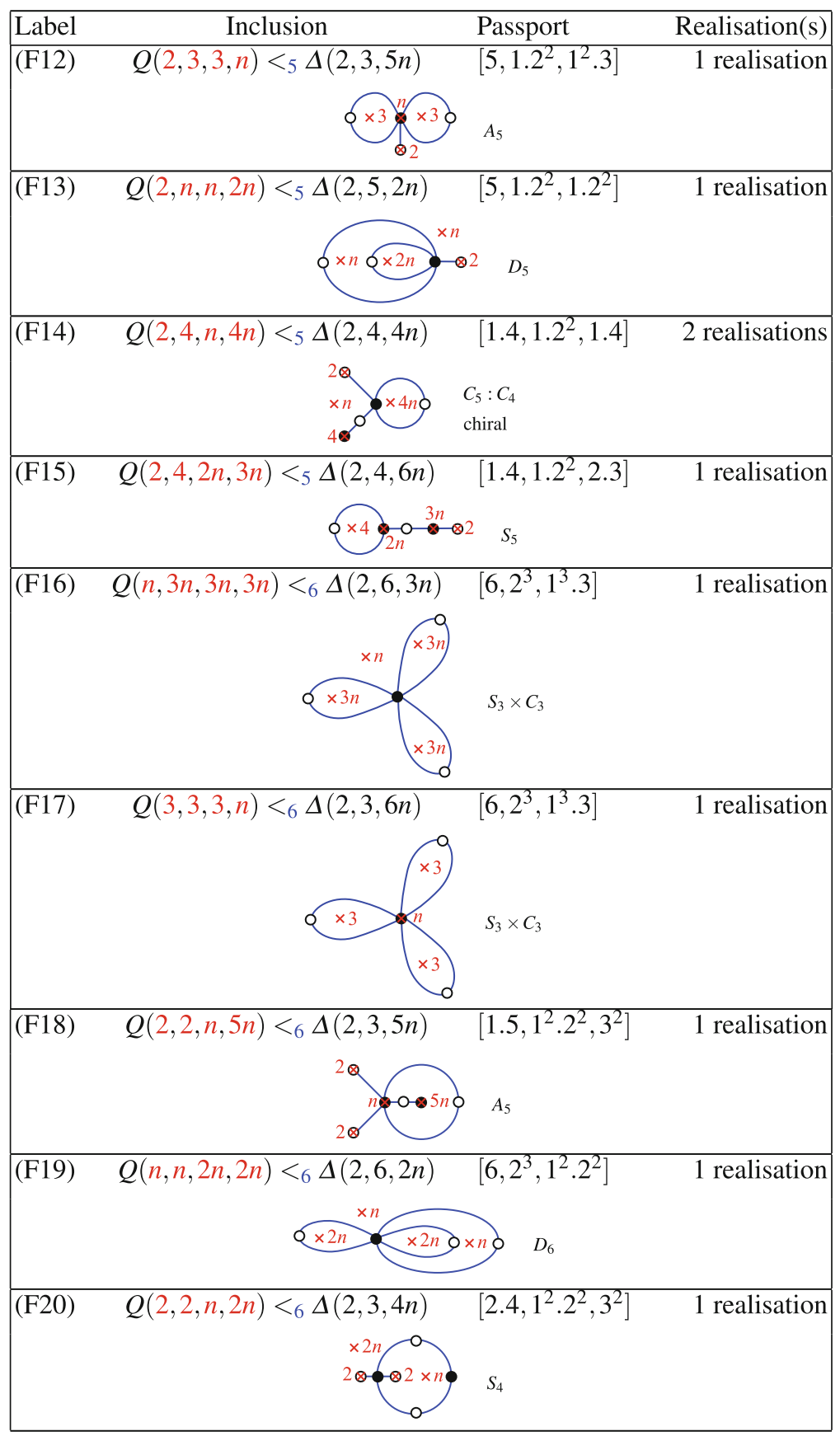


Table 5 (continued)

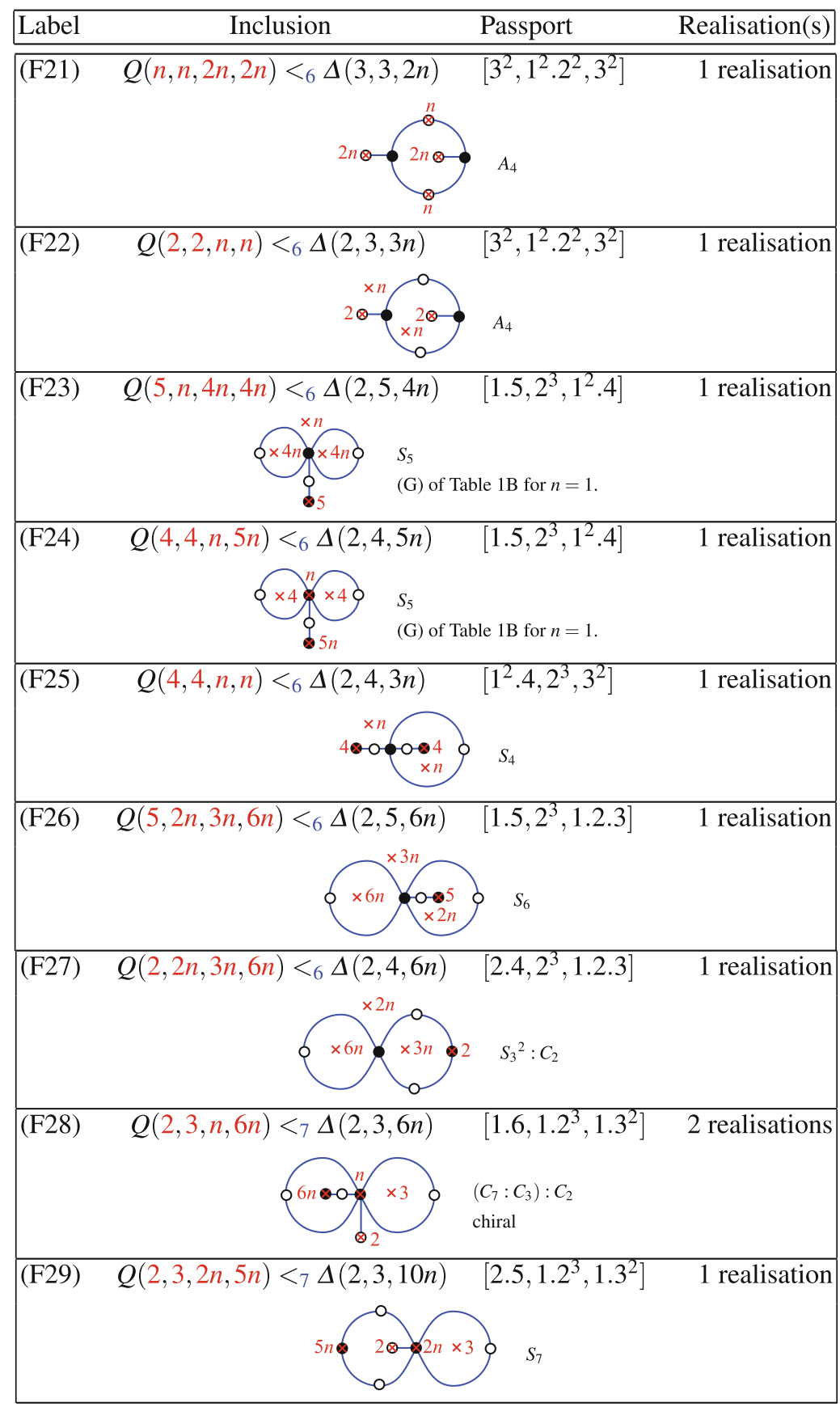


Table 5 (continued)

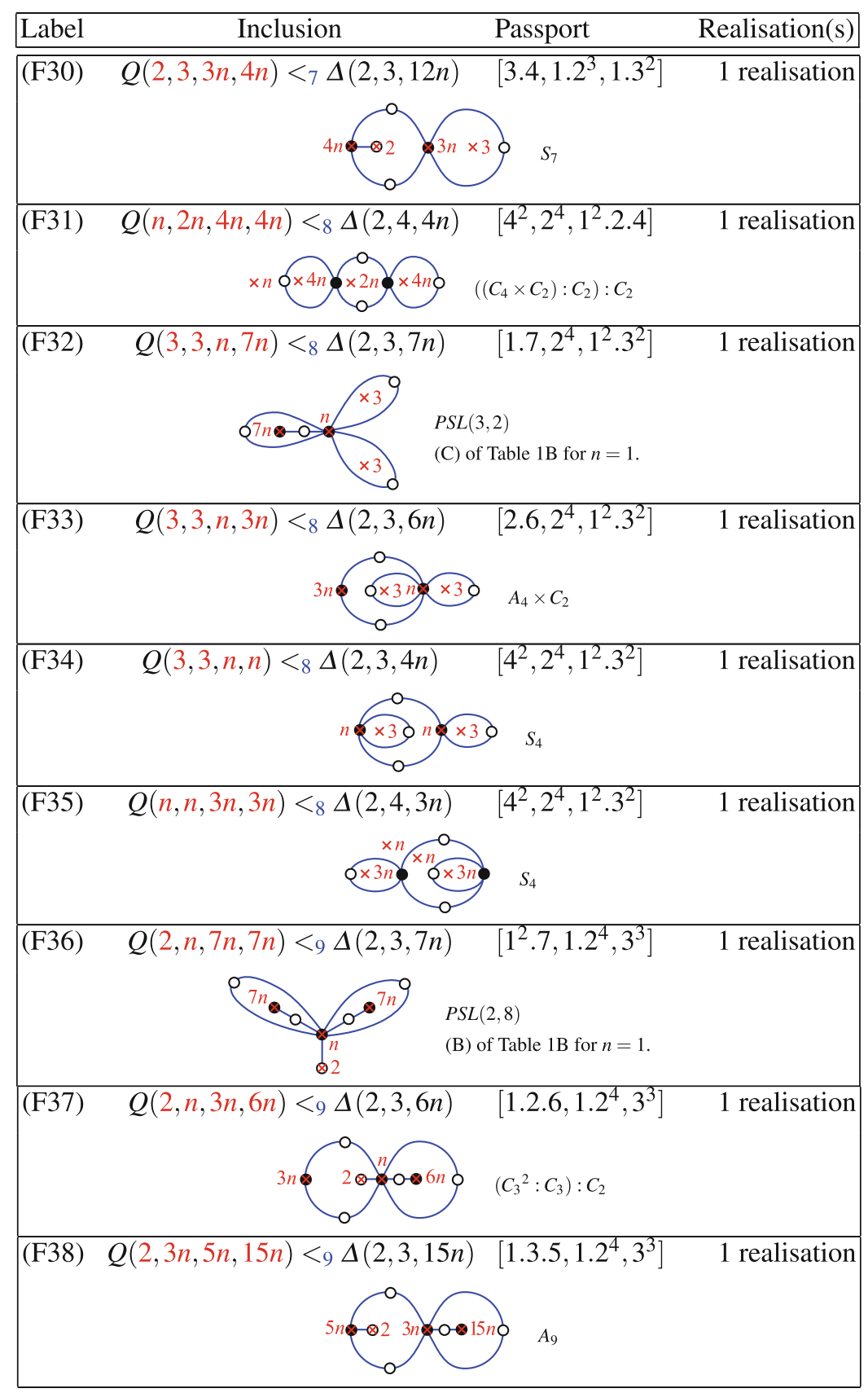


Table 5 (continued)

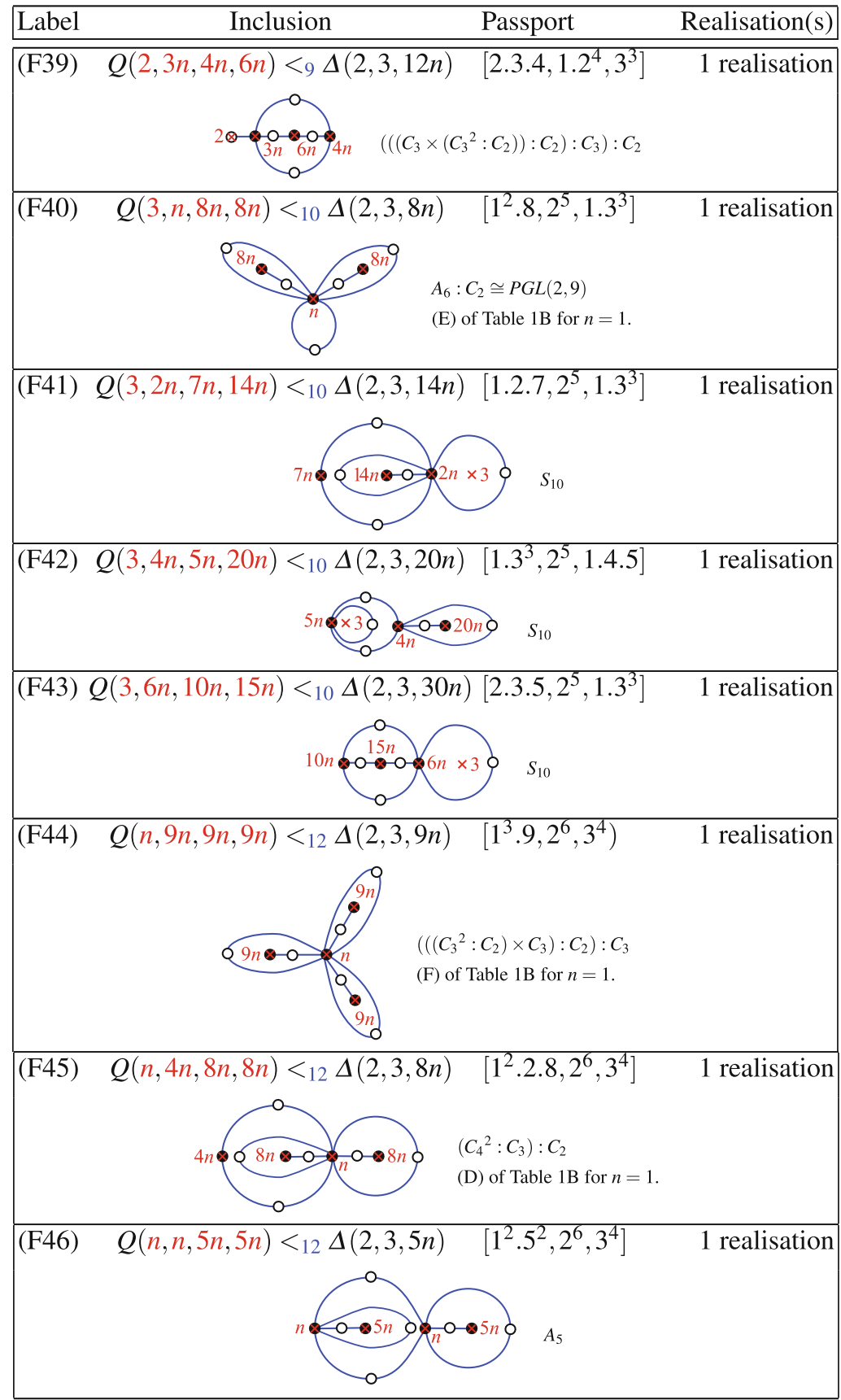


Table 5 (continued)

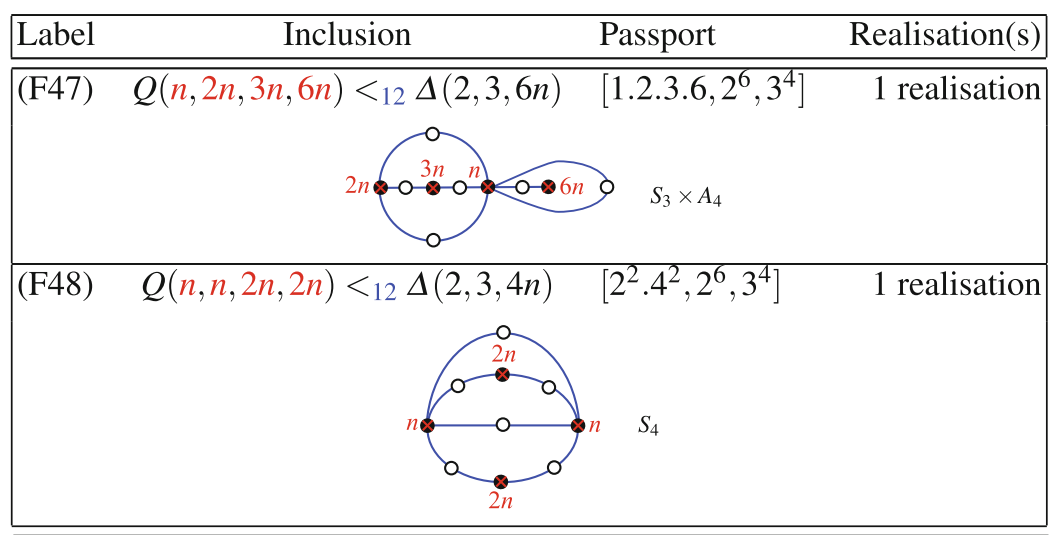

Table 6 Sporadic non-normal inclusions of quadrangle groups in triangle groups. (There are no sporadic normal inclusions)

\begin{tabular}{|c|c|c|}
\hline Label & Passport & Realisation(s) \\
\hline (S1) & $\begin{aligned} Q(2,2,2,4)<{ }_{5} \Delta(2,4,5) & {\left[5,1^{3} .2,1.4\right] } \\
2 \otimes & \\
2 \otimes \times 4 & S_{5}\end{aligned}$ & 1 realisation \\
\hline (S2) & $\begin{array}{c}Q(3,3,3,3)<_{5} \Delta(3,3,5) \quad\left[5,1^{2} .3,1^{2} .3\right] \\
30 \times 3 \times A_{5}\end{array}$ & 1 realisation \\
\hline (S3) & $\begin{array}{c}Q(3,3,4,4)<_{5} \Delta(3,4,4) \quad\left[1.4,1.4,1^{2} .3\right] \\
4 \otimes \cdot \frac{\times 3}{\times 3} \bigcirc \bullet 4 S_{5}\end{array}$ & 1 realisation \\
\hline (S5) & $\begin{array}{l}Q(3,3,3,5)<_{6} \Delta(3,3,5)\left[1^{3} \cdot 3,1.5,3^{2}\right] \\
Q(2,3,3,3)<_{6} \Delta(3,3,4) \quad\left[1^{3} \cdot 3,2.4,3^{2}\right]\end{array}$ & 1 realisation \\
\hline (S6) & 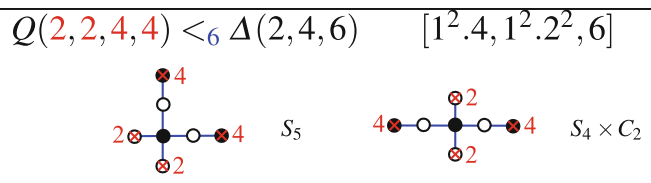 & 2 realisations \\
\hline
\end{tabular}


Table 6 (continued)

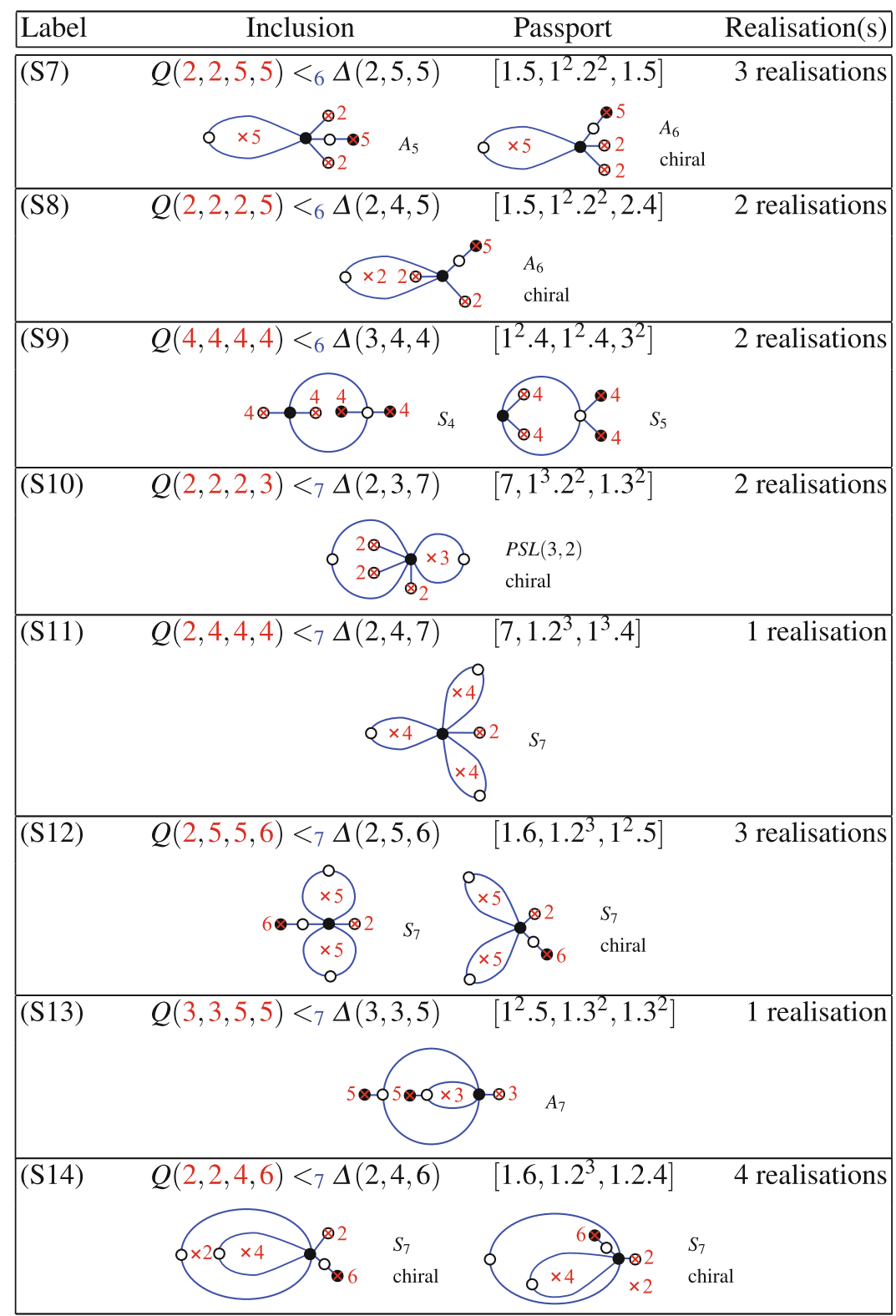


Table 6 (continued)

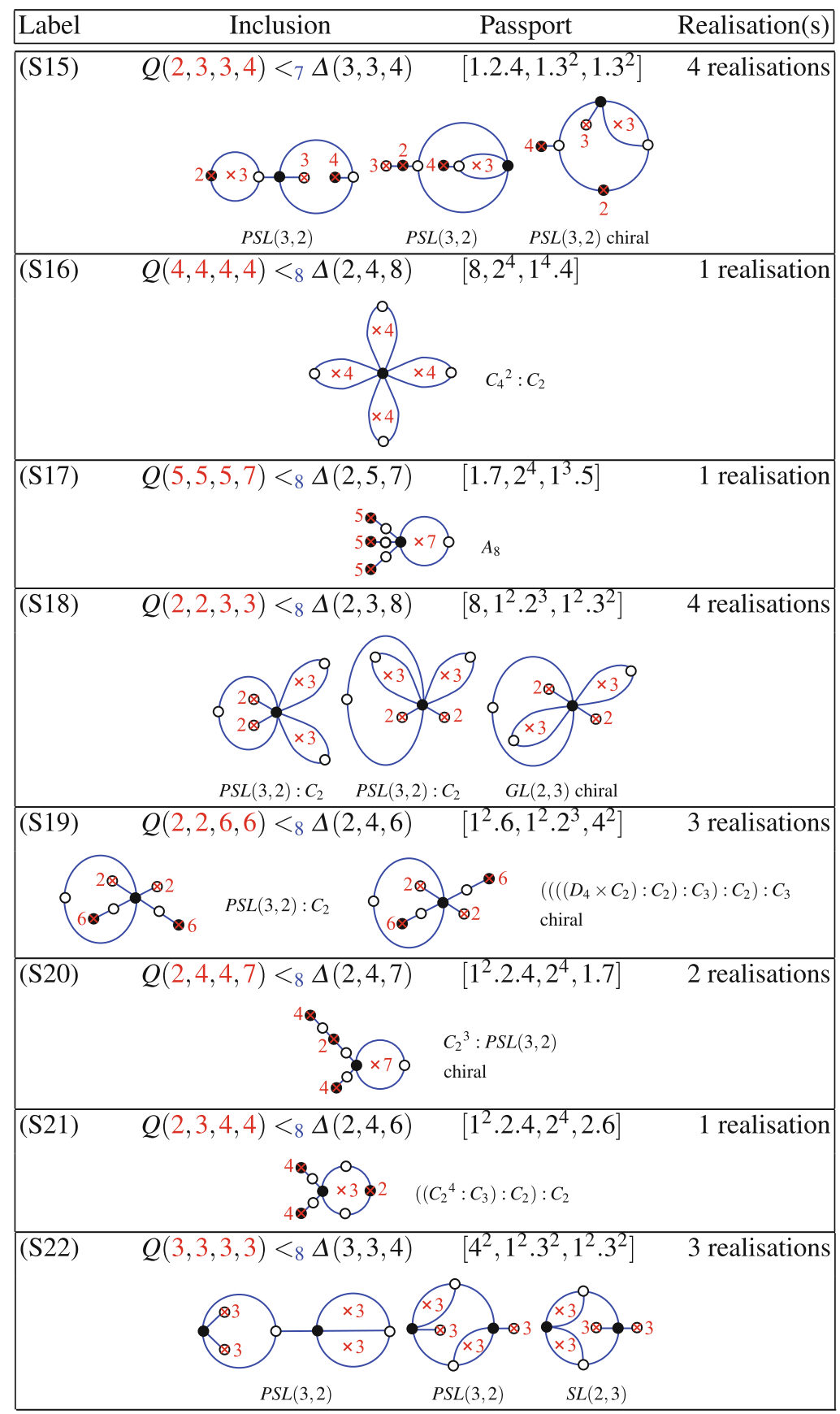


Table 6 (continued)

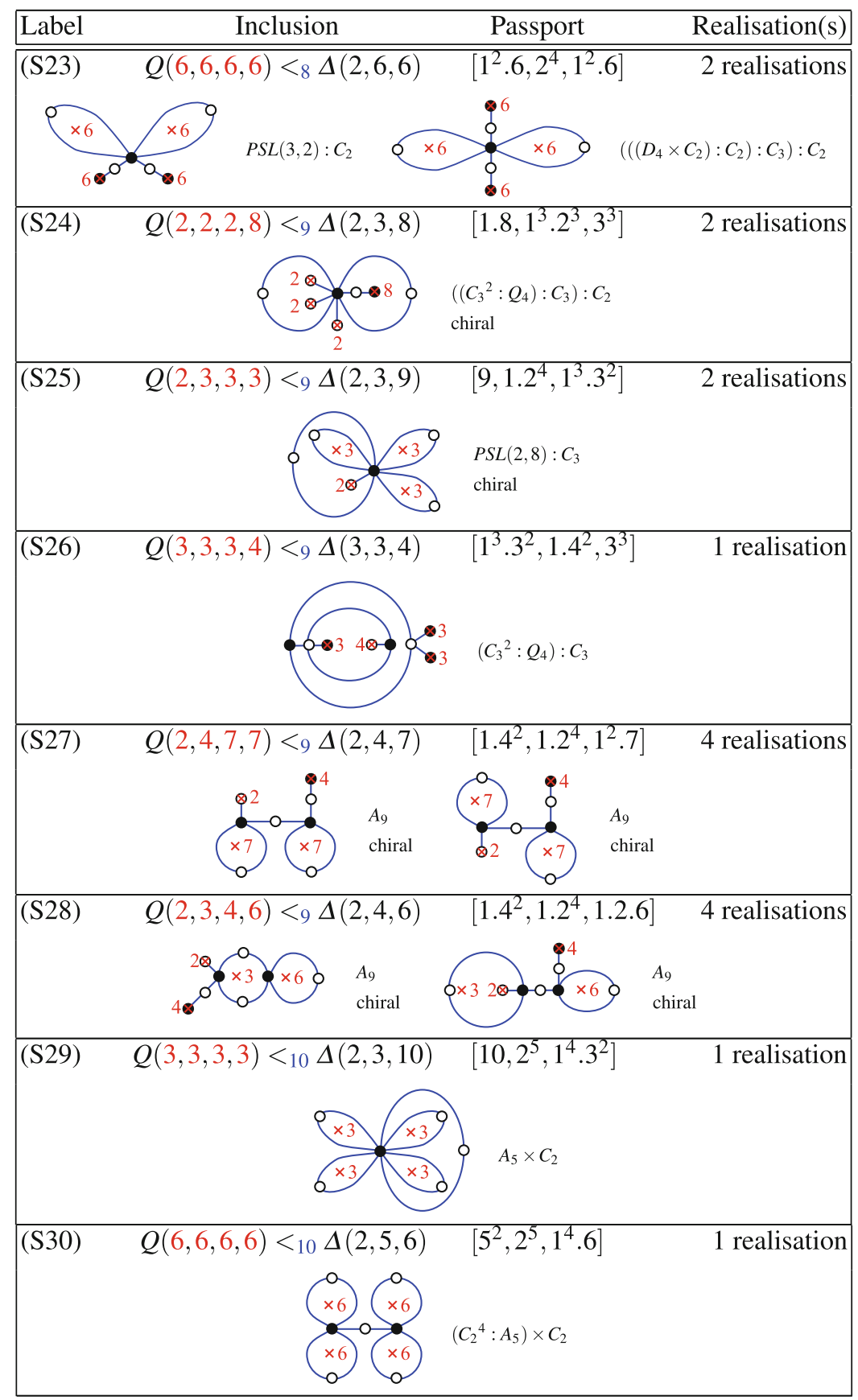


Table 6 (continued)

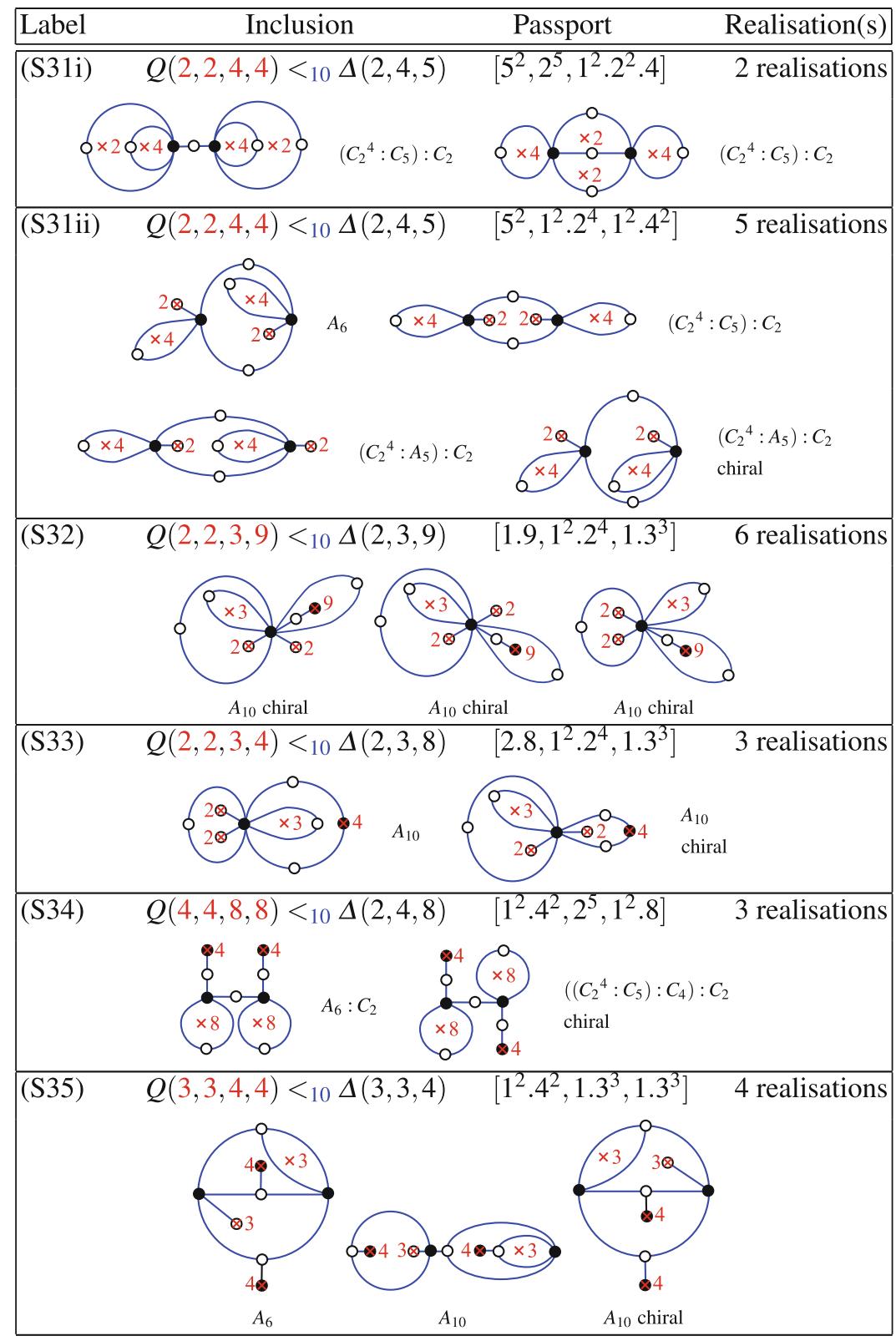

(continued) 
Table 6 (continued)

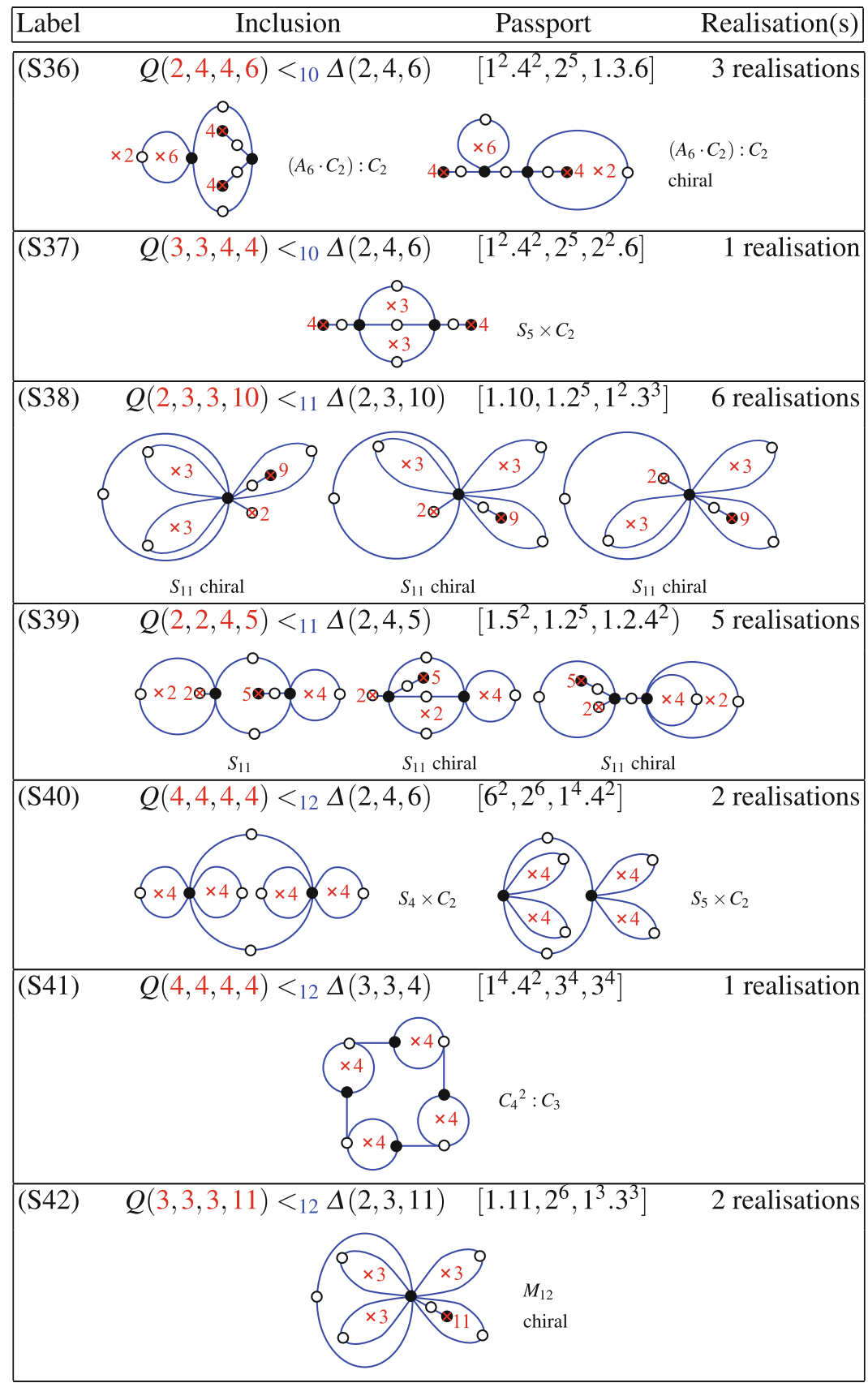


Table 6 (continued)

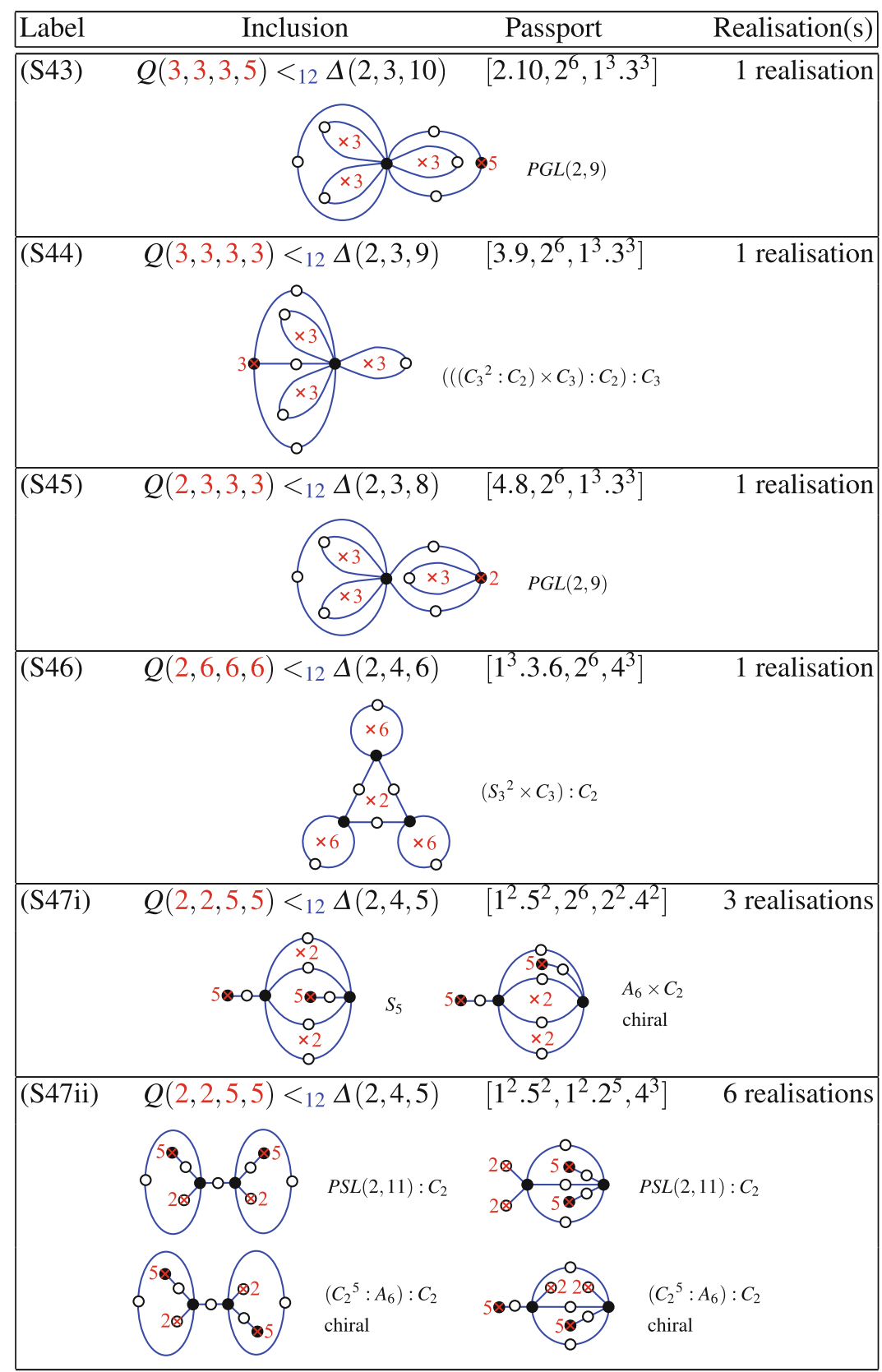


Table 6 (continued)

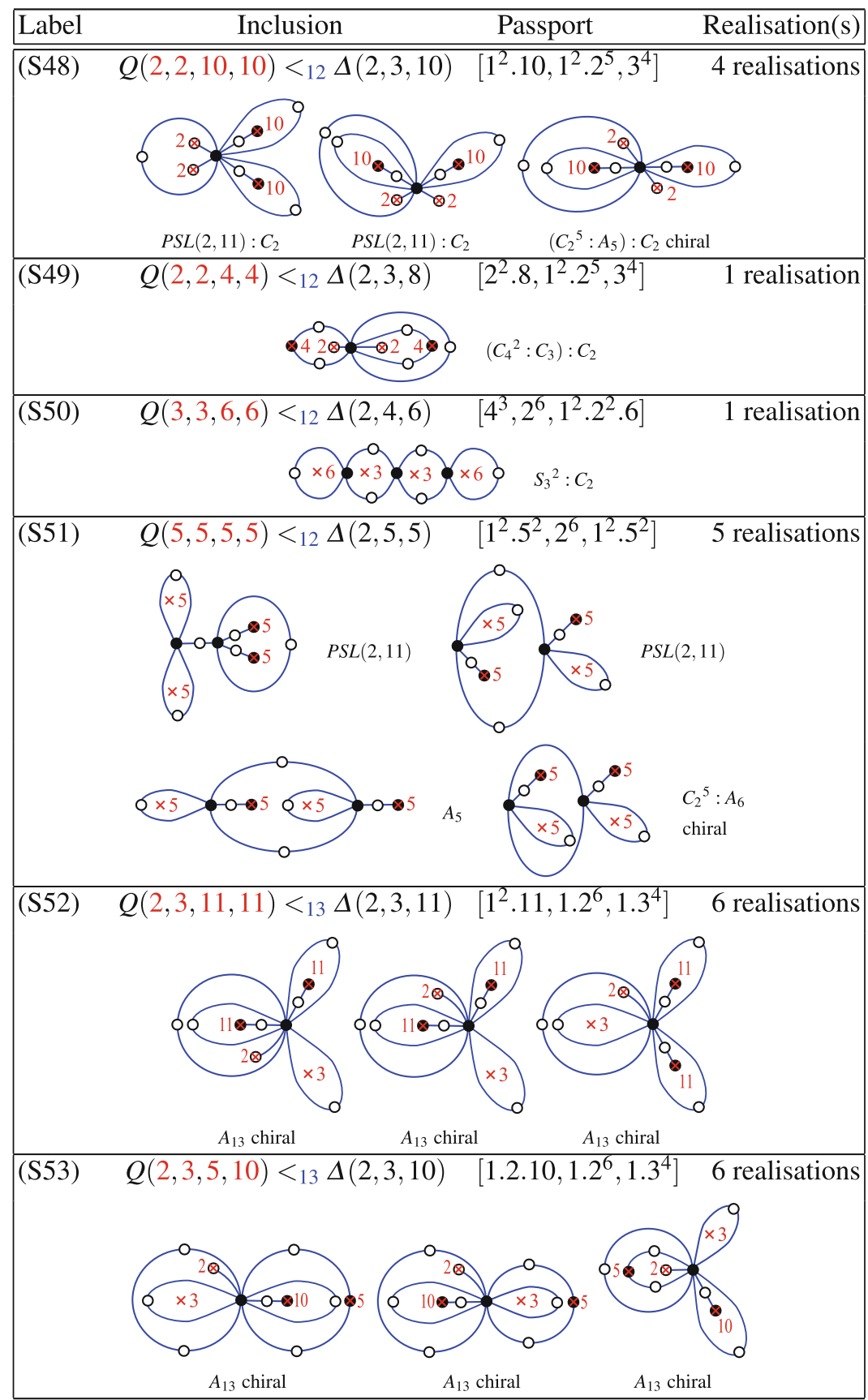

(continued) 
Table 6 (continued)

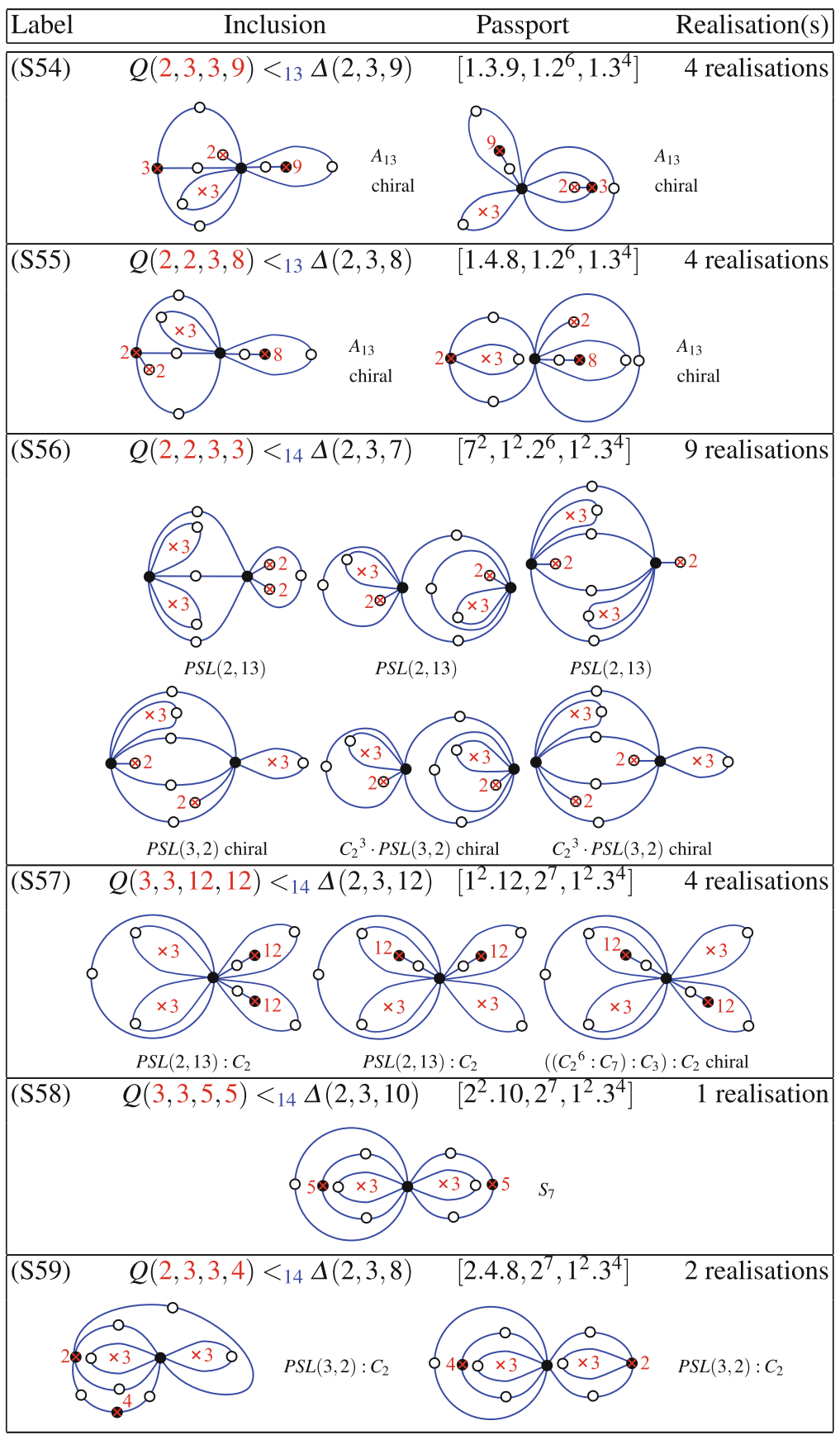

(continued) 
Table 6 (continued)

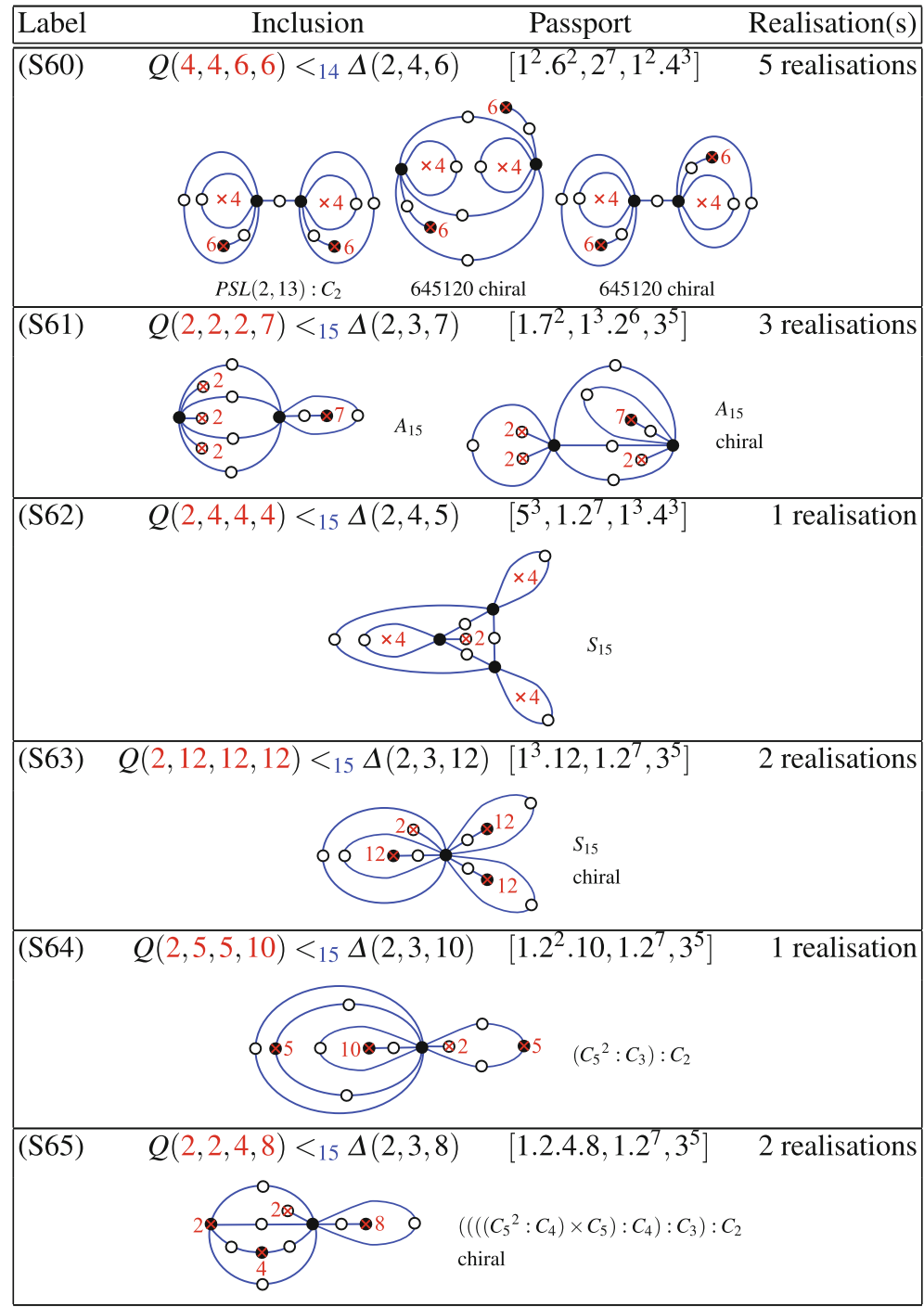

(continued) 
Table 6 (continued)

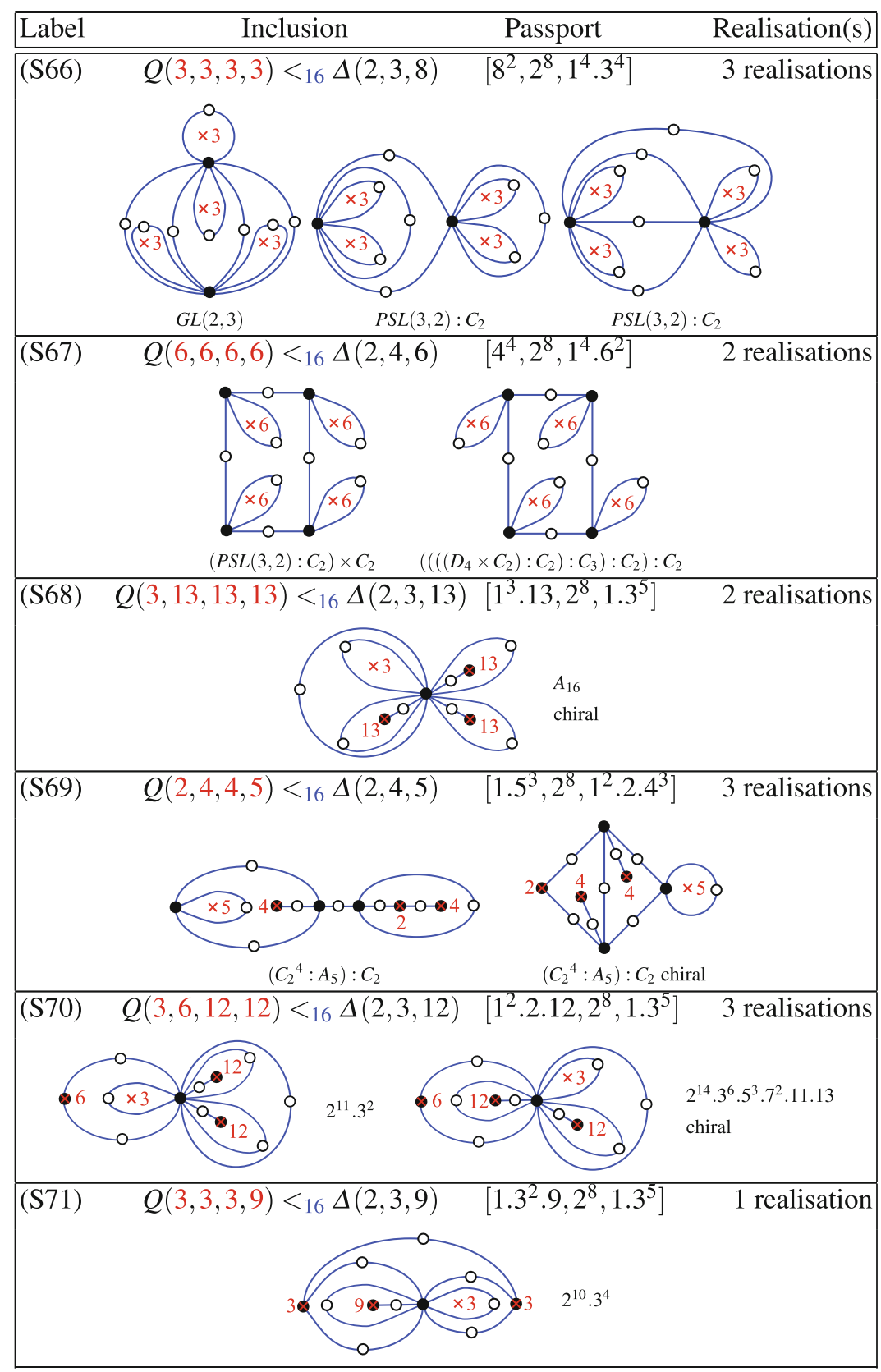

(continued) 
Table 6 (continued)

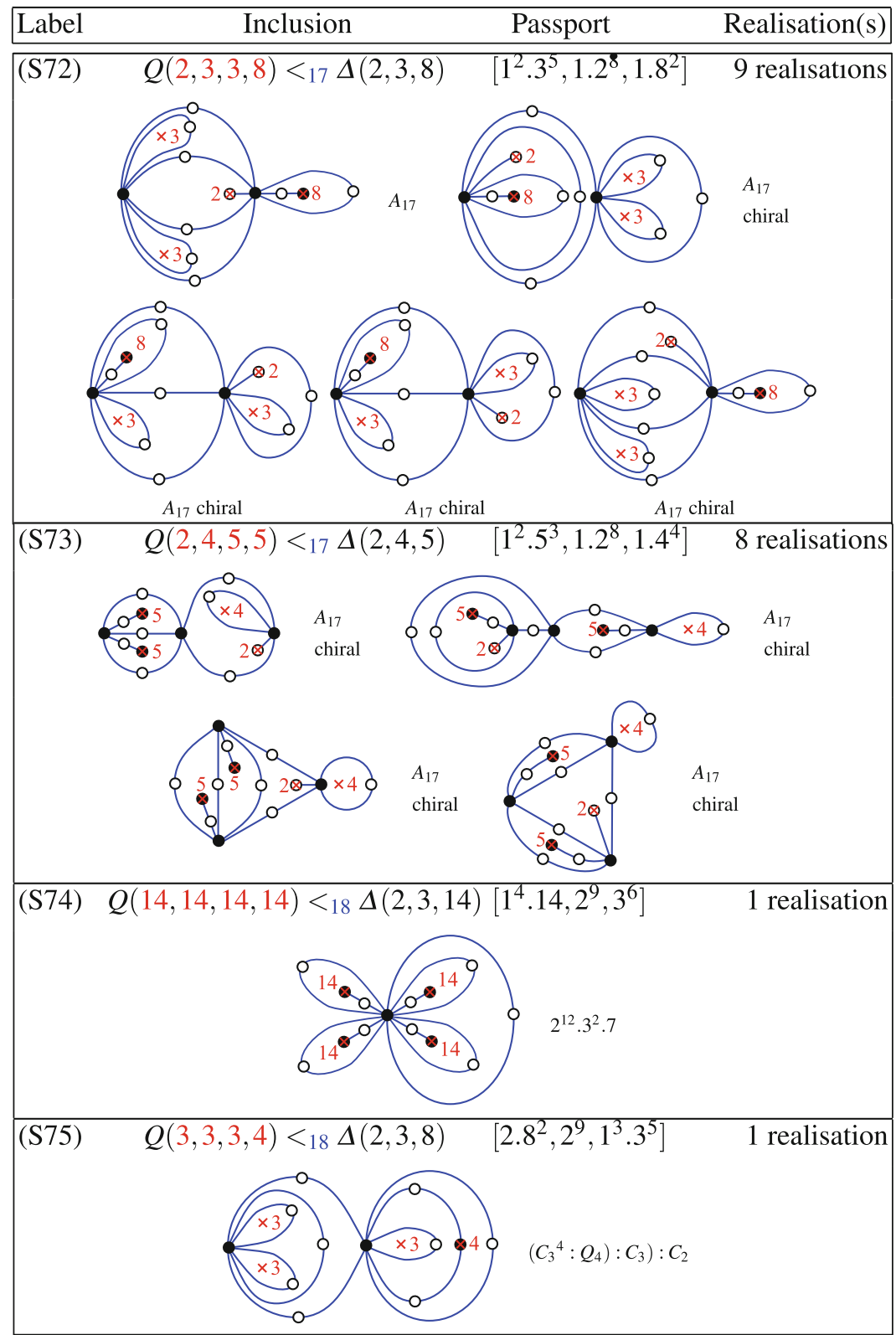

(continued) 
Table 6 (continued)

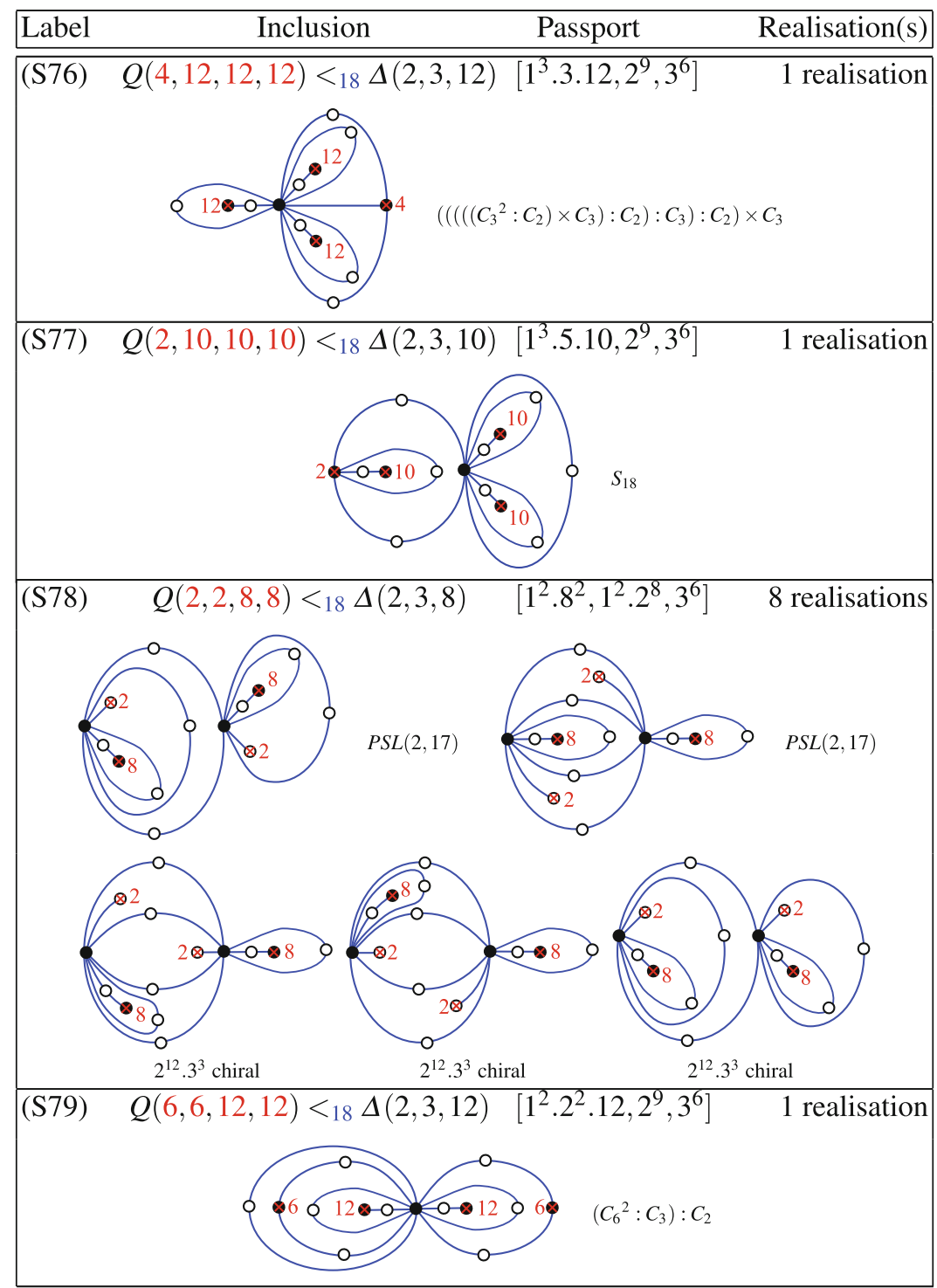

(continued) 
Table 6 (continued)

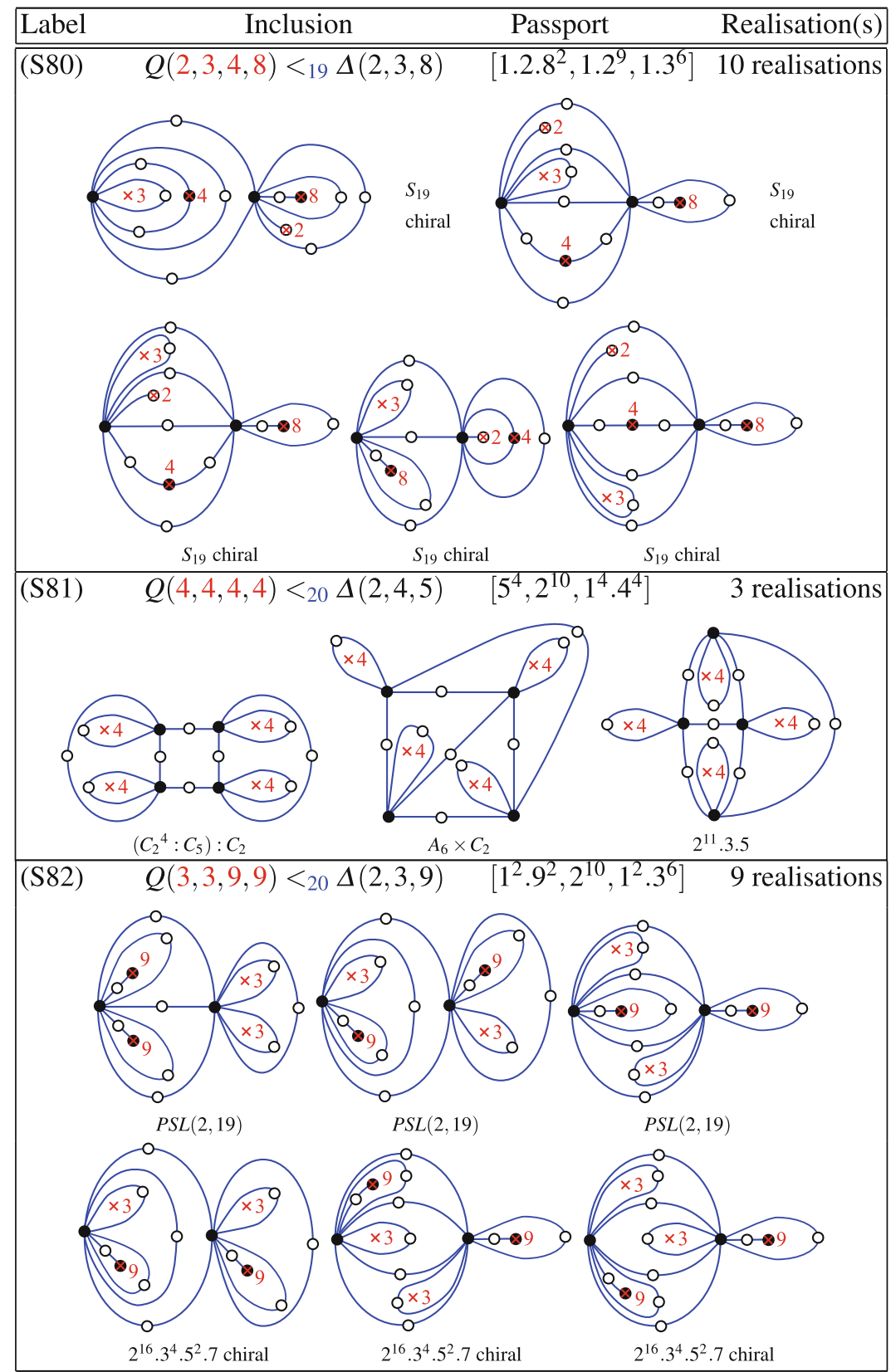

(continued) 
Table 6 (continued)

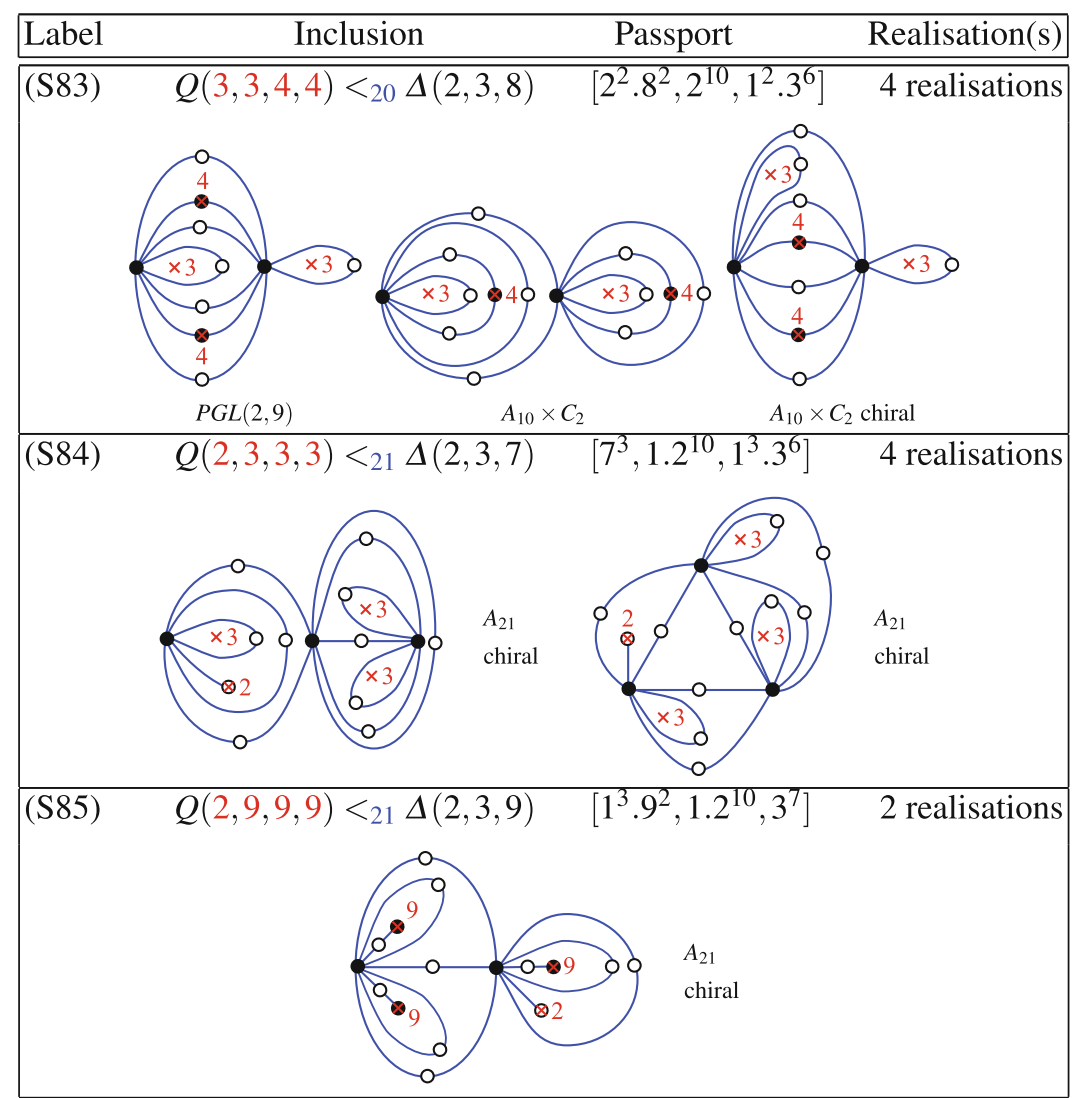

(continued) 
Table 6 (continued)

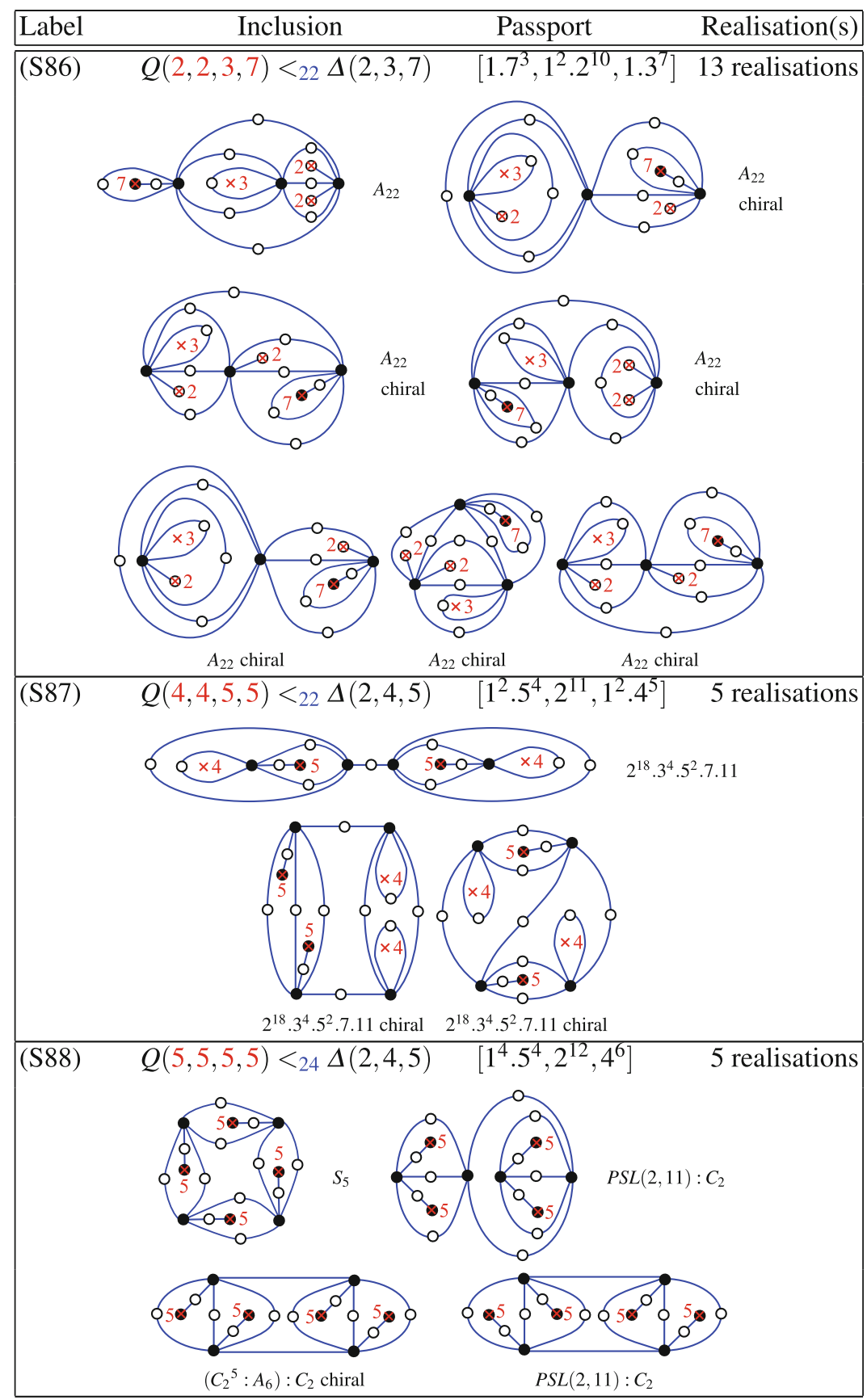

(continued) 
Table 6 (continued)

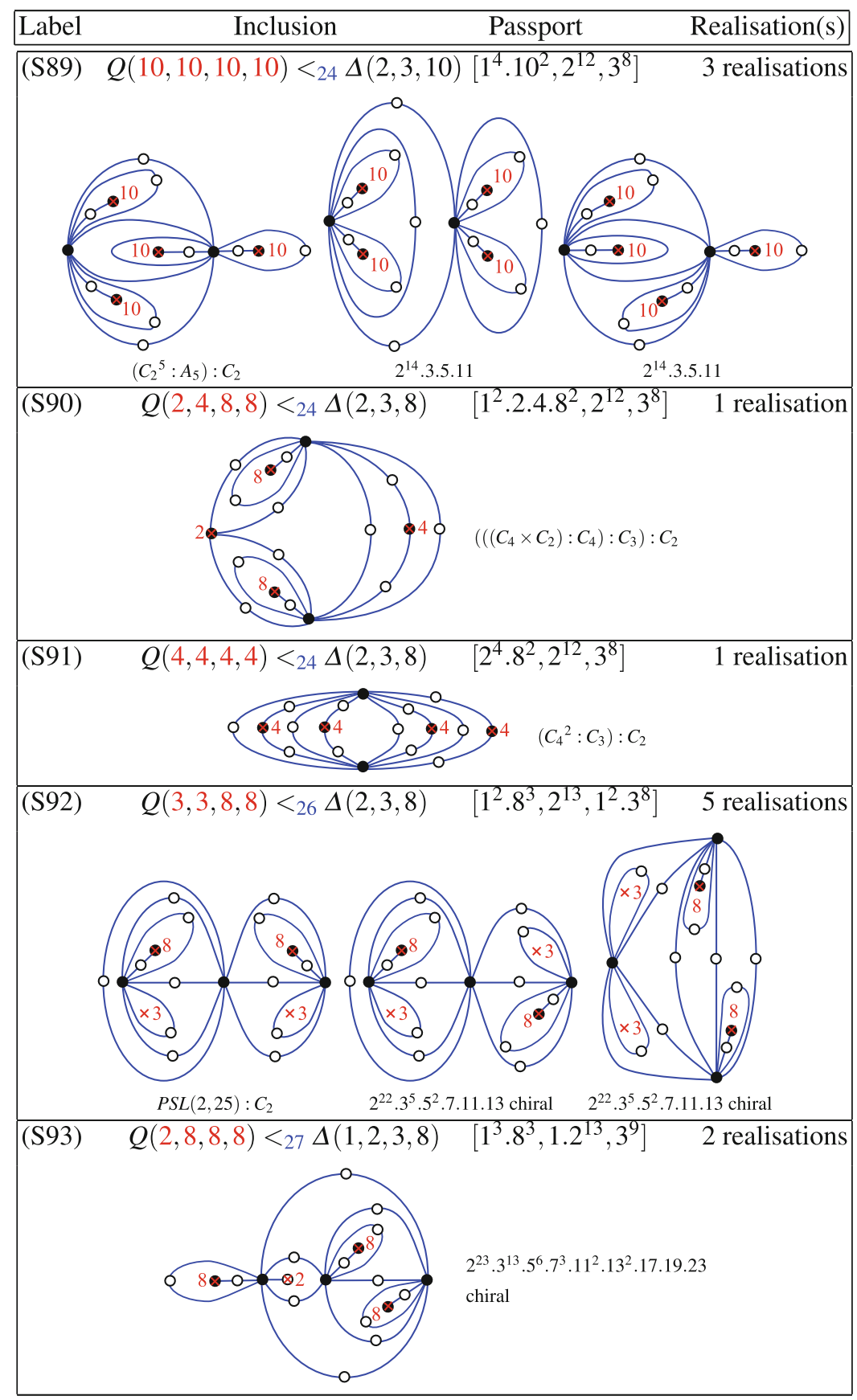


Table 6 (continued)

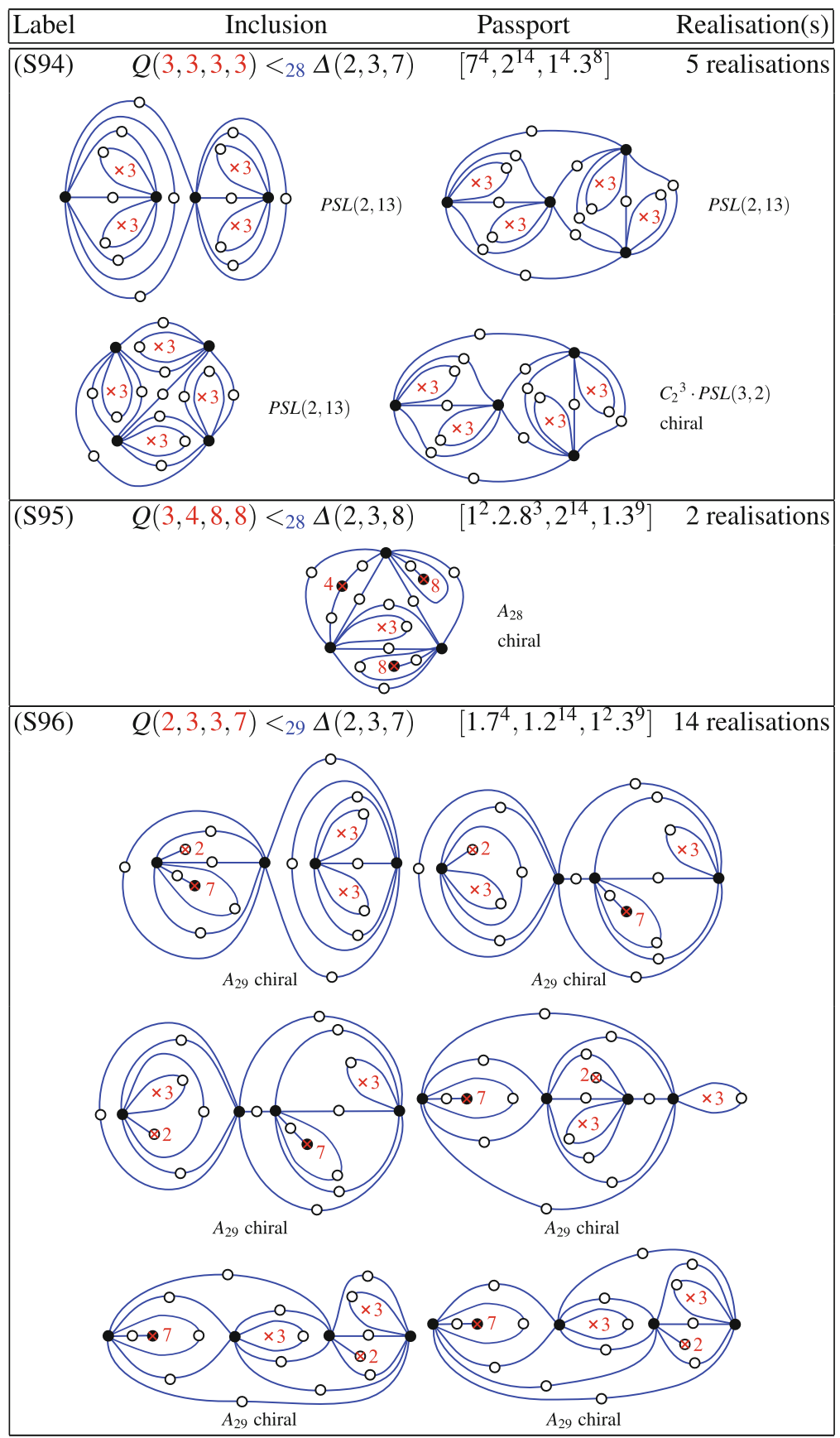

(continued) 
Table 6 (continued)

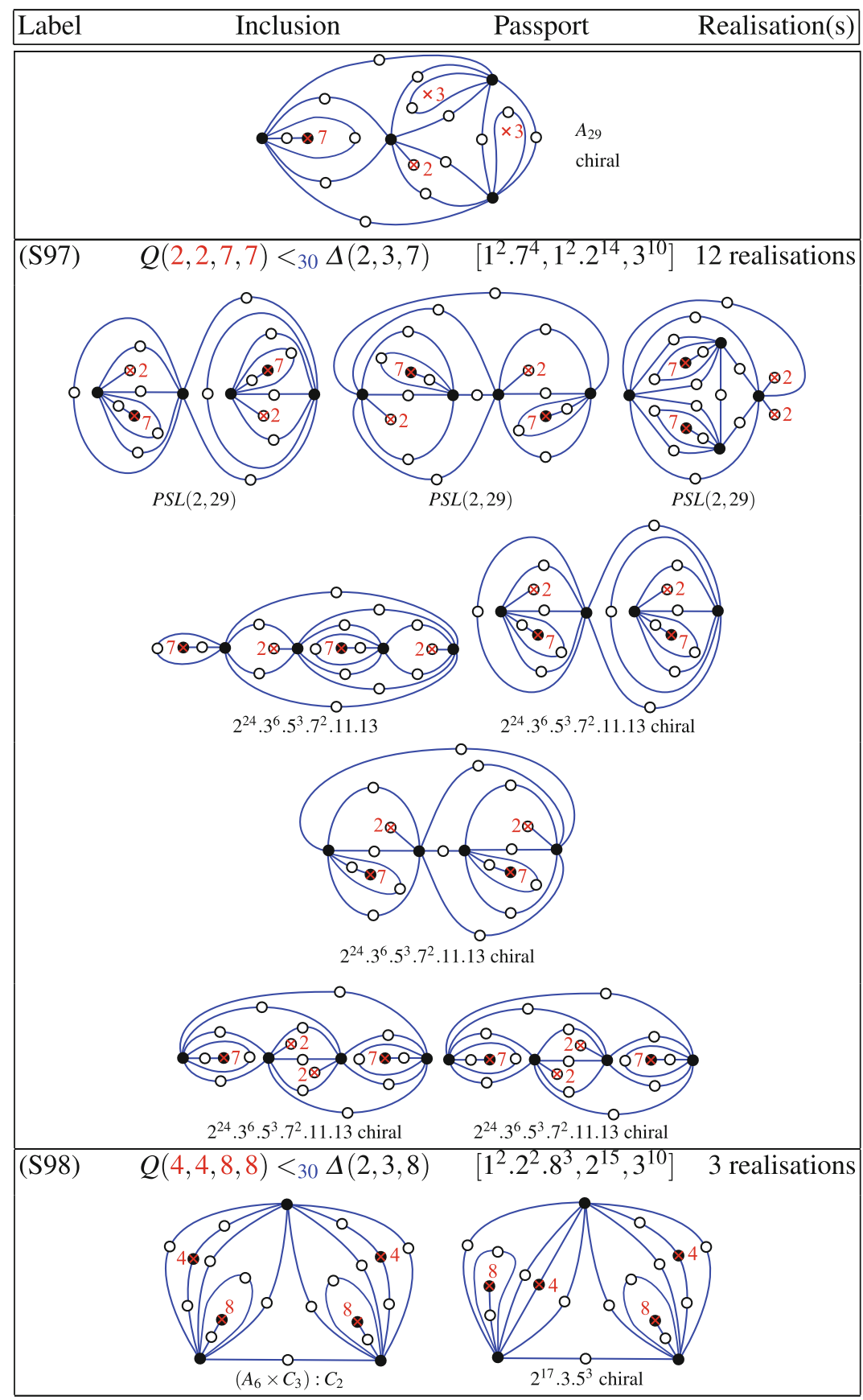

(continued) 
Table 6 (continued)

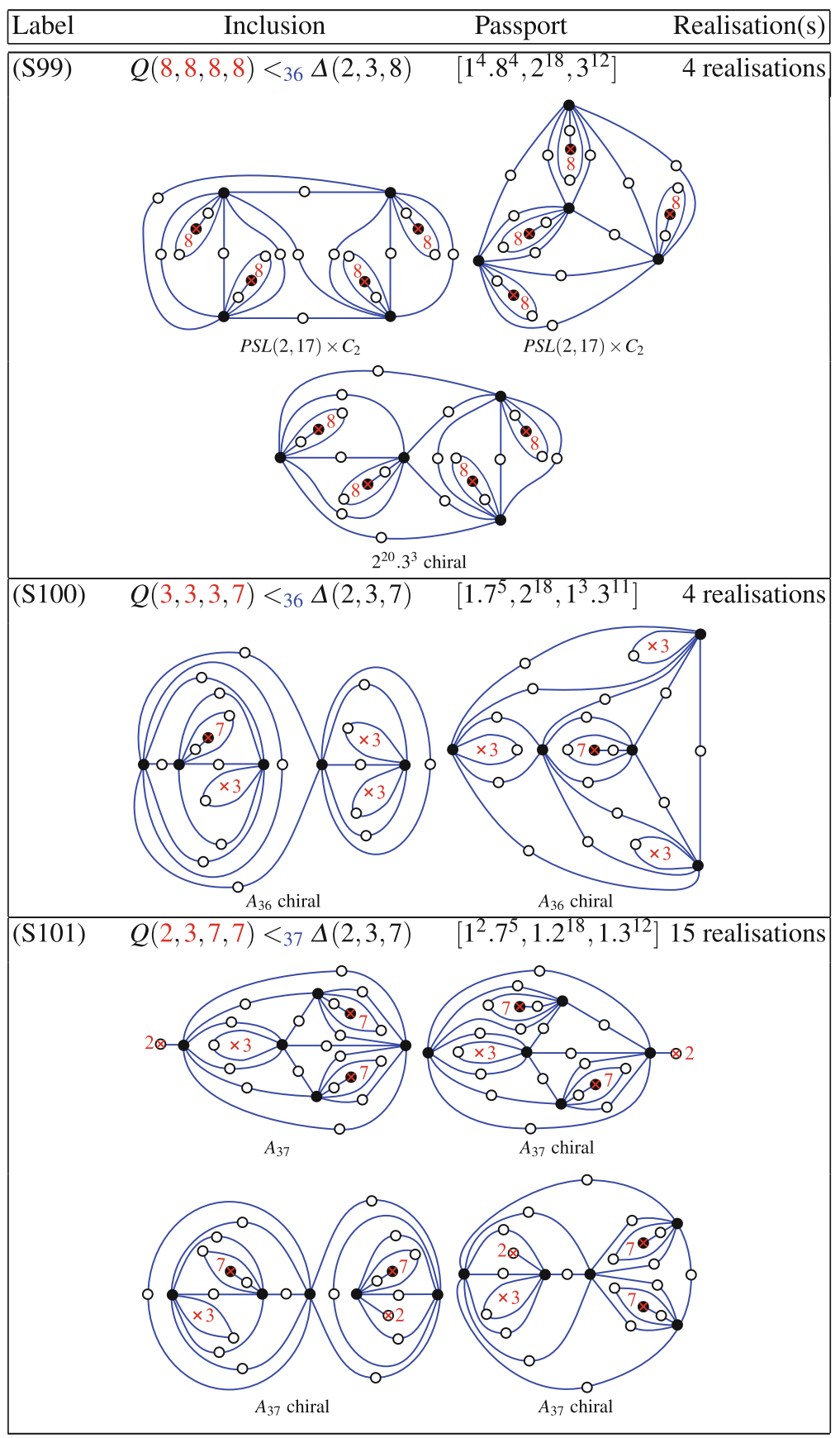

(continued) 
Table 6 (continued)

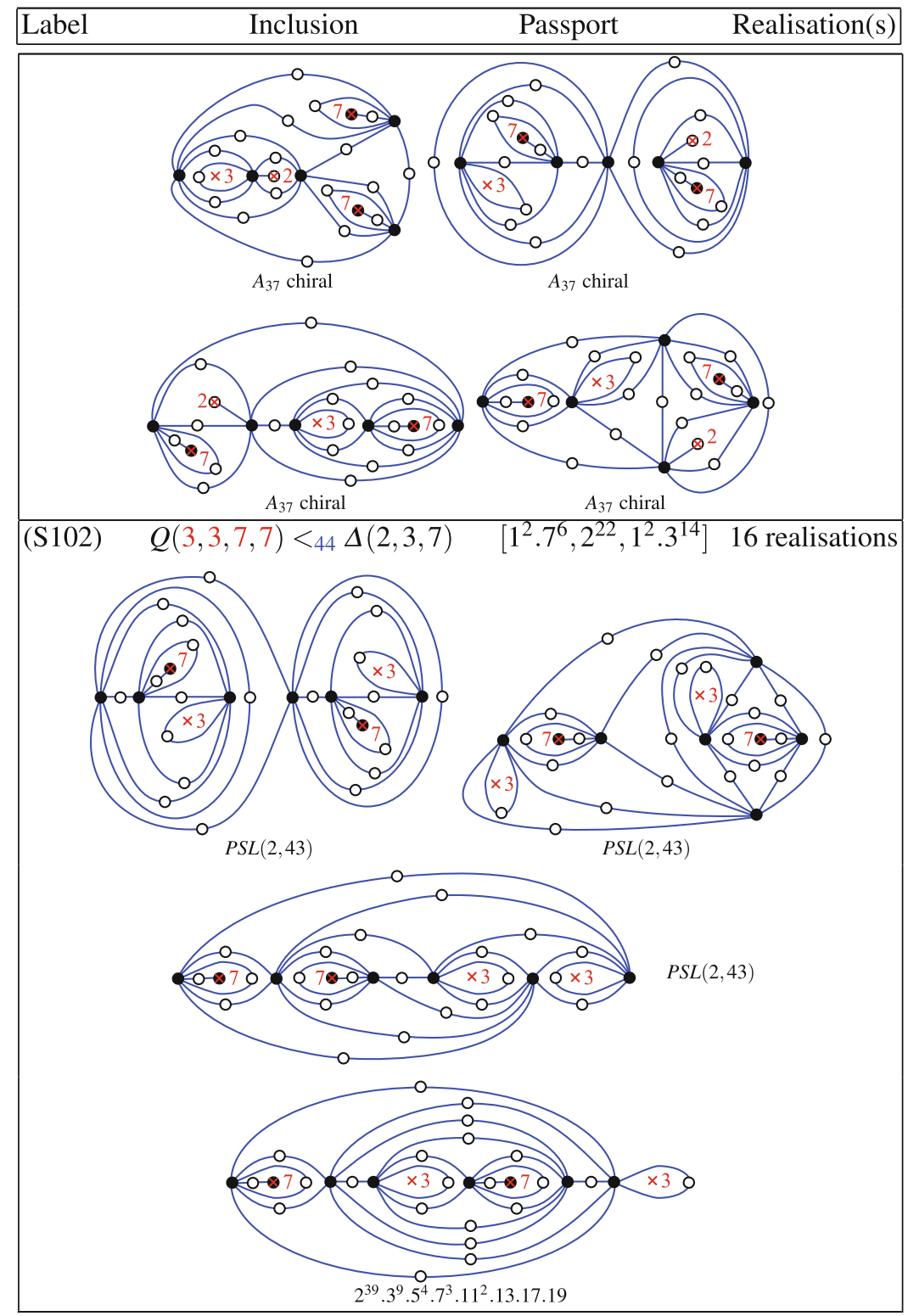

(continued) 
Table 6 (continued)

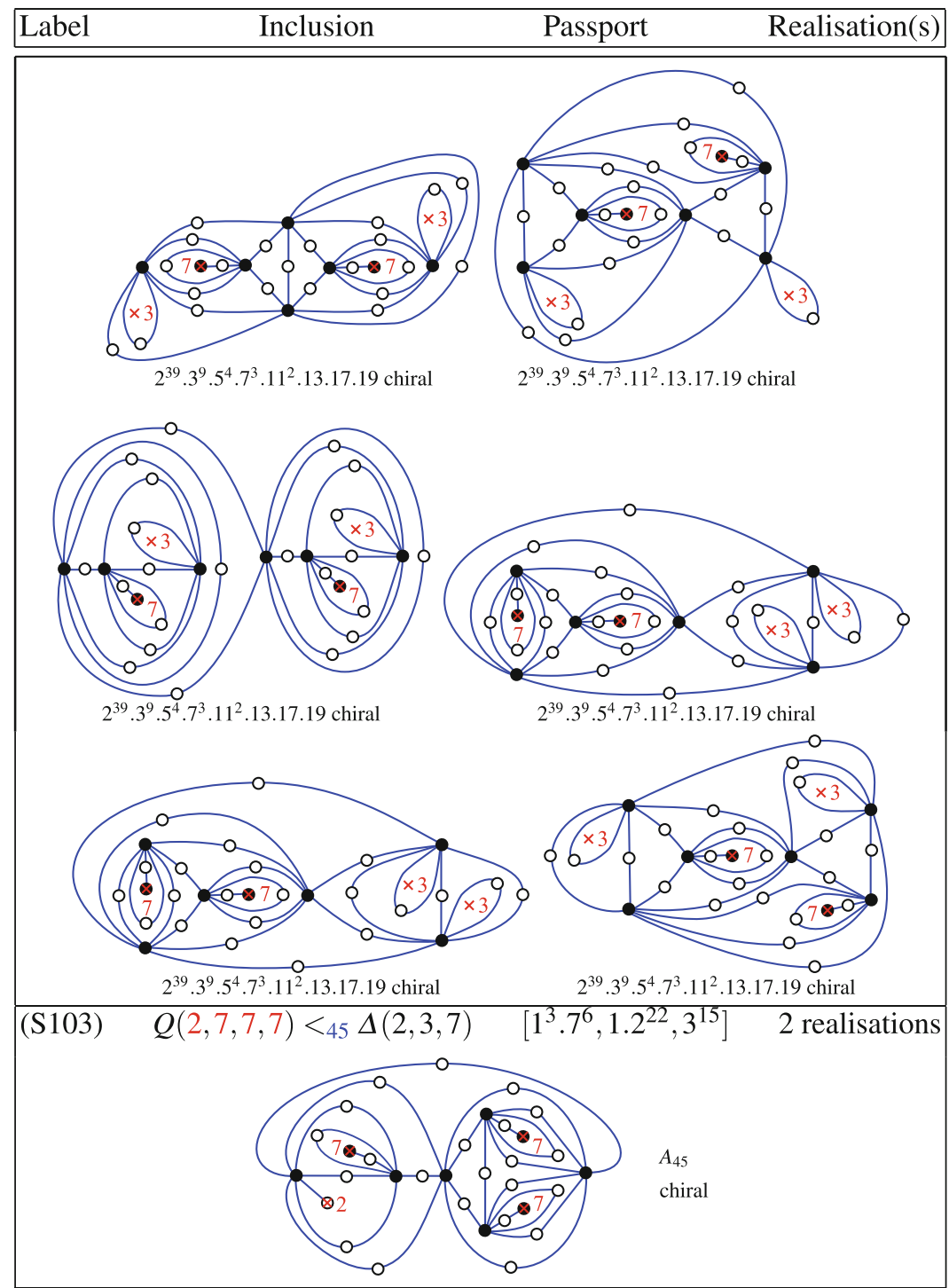

(continued) 
Table 6 (continued)

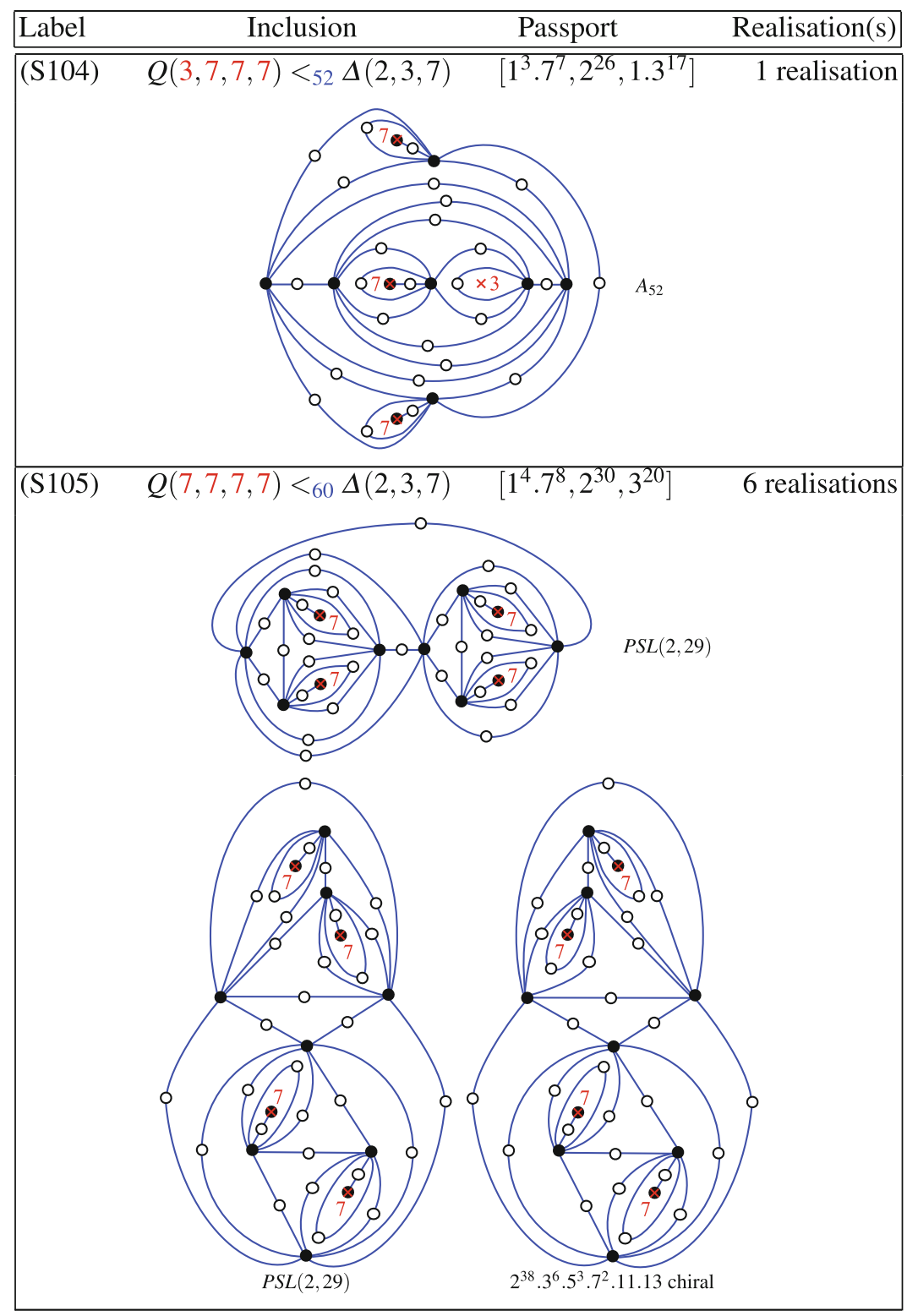

(continued) 
Table 6 (continued)

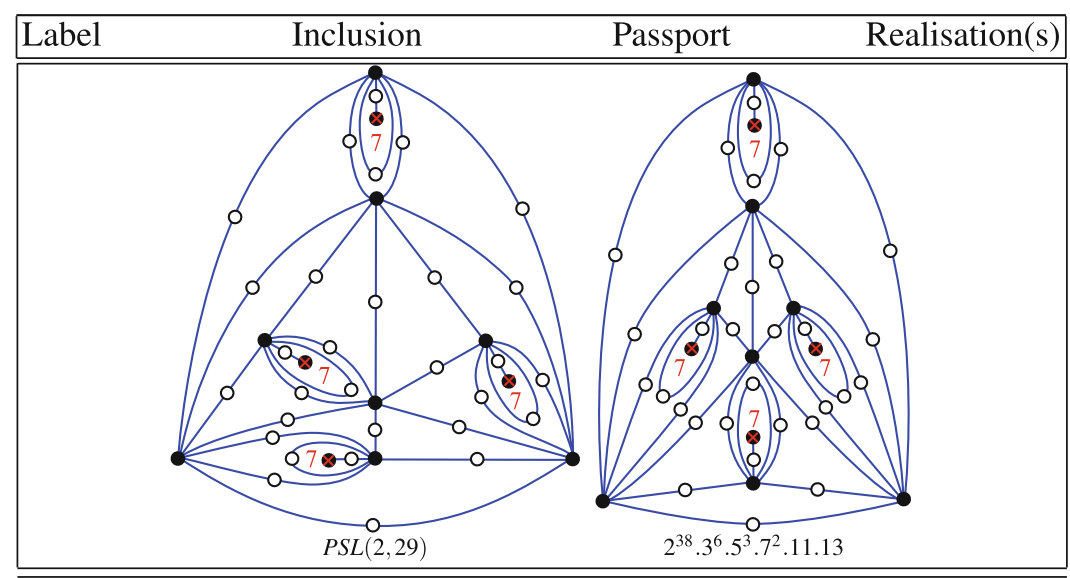




\section{Numerical Solutions with No Corresponding Inclusions}

See Table 7.

Table 7 Admissible passports for which there is no inclusion (Forbidden passports)

\begin{tabular}{|c|c|c|}
\hline Numerical solution & Passport & Notes \\
\hline $\begin{array}{l}(m, 3 m, n, n)<_{4} \\
(1,2,3 m, 2 n)\end{array}$ & {$\left[1.3,2^{2}, 2^{2}\right]$} & $\begin{array}{l}m=1: \Delta(3, n, n) \nless \\
\Delta(2,3,2 n)\end{array}$ \\
\hline $\begin{array}{l}(n, 3 n, 3 n, 3 n)<6 \\
(1,3,3,3 n)\end{array}$ & {$\left[1^{3} .3,3^{2}, 3^{2}\right]$} & \\
\hline$(2, n, 4 n, 4 n)<_{6}(1,2,4,4 n)$ & {$\left[1^{2} .4,2^{3}, 2.4\right]$} & \\
\hline$(4,4, n, 2 n)<_{6}(1,2,4,4 n)$ & {$\left[1^{2} .4,2^{3}, 2.4\right]$} & \\
\hline$(1,2 n, 3 n, 6 n)<_{6}(1,2,3,6 n)$ & {$\left[1.2 .3,2^{3}, 3^{2}\right]$} & \\
\hline$(5, n, n, n)<_{6}(1,2,5,2 n)$ & {$\left[1.5,2^{3}, 2^{3}\right]$} & \\
\hline$(2, n, n, n)<_{6}(1,2,4,2 n)$ & {$\left[2^{3}, 2^{3}, 2.4\right]$} & \\
\hline $\begin{array}{l}(n, 5 n, 5 n, 5 n)<8 \\
(1,2,4,5 n)\end{array}$ & {$\left[1^{3} .5,2^{4}, 4^{2}\right]$} & $\begin{array}{l}n=1: \Delta(5,5,5) \nless \\
\Delta(2,4,5)\end{array}$ \\
\hline$(3,3,3 n, 5 n)<8(1,2,3,15 n)$ & {$\left[3.5,2^{4}, 1^{2} \cdot 3^{2}\right]$} & \\
\hline $\begin{array}{l}(2 n, 3 n, 3 n, 6 n)<8 \\
(1,2,4,6 n)\end{array}$ & {$\left[1.2^{2} .3,2^{4}, 4^{2}\right]$} & \\
\hline$(2, n, n, 4 n)<9(1,2,3,4 n)$ & {$\left[1.4^{2}, 1.2^{4}, 3^{3}\right]$} & \\
\hline $\begin{array}{l}(2,2 n, 5 n, 5 n)<9 \\
(1,2,3,10 n)\end{array}$ & {$\left[2^{2} .5,1.2^{4}, 3^{3}\right]$} & \\
\hline$(2, n, n, n)<9(1,2,3,3 n)$ & {$\left[3^{3}, 1.2^{4}, 3^{3}\right]$} & \\
\hline$(3, n, 2 n, 6 n)<10(1,2,3,6 n)$ & {$\left[1.3 .6,2^{5}, 1.3^{3}\right]$} & \\
\hline$(3, n, 3 n, 3 n)<10(1,2,3,6 n)$ & {$\left[2^{2} .6,2^{5}, 1.3^{3}\right]$} & \\
\hline$(3, n, n, 2 n)<10(1,2,3,4 n)$ & {$\left[2.4^{2}, 2^{5}, 1.3^{3}\right]$} & \\
\hline $\begin{array}{l}(3,3 n, 4 n, 4 n)<10 \\
(1,2,3,12 n)\end{array}$ & {$\left[3^{2} .4,2^{5}, 1.3^{3}\right]$} & \\
\hline $\begin{array}{l}(3 n, 7 n, 21 n, 21 n)<12 \\
(1,2,3,21 n)\end{array}$ & {$\left[1^{2} \cdot 3 \cdot 7,2^{6}, 3^{4}\right]$} & \\
\hline $\begin{array}{l}(2 n, 3 n, 12 n, 12 n)<12 \\
(1,2,3,12 n)\end{array}$ & {$\left[1^{2} .4 .6,2^{6}, 3^{4}\right]$} & \\
\hline $\begin{array}{l}(2 n, 7 n, 7 n, 14 n)<12 \\
(1,2,3,14 n)\end{array}$ & {$\left[1.2^{2} .7,2^{6}, 3^{4}\right]$} & \\
\hline $\begin{array}{l}(4 n, 5 n, 10 n, 20 n)<12 \\
(1,2,3,20 n)\end{array}$ & {$\left[1.2 .4 .5,2^{6}, 3^{4}\right]$} & \\
\hline $\begin{array}{l}(3 n, 5 n, 5 n, 15 n)<12 \\
(1,2,3,15 n)\end{array}$ & {$\left[1.3^{2} .5,2^{6}, 3^{4}\right]$} & \\
\hline $\begin{array}{l}(3 n, 3 n, 4 n, 12 n)<12 \\
(1,2,3,12 n)\end{array}$ & {$\left[1.3 .4^{2}, 2^{6}, 3^{4}\right]$} & \\
\hline
\end{tabular}


Table 7 (continued)

\begin{tabular}{|c|c|c|}
\hline Numerical solution & Passport & Notes \\
\hline $\begin{array}{l}(n, 3 n, 3 n, 3 n)<12 \\
(1,2,3,6 n)\end{array}$ & {$\left[2^{3} \cdot 6,2^{6}, 3^{4}\right]$} & \\
\hline $\begin{array}{l}(6 n, 10 n, 15 n, 15 n)<12 \\
(1,2,3,30 n)\end{array}$ & {$\left[2^{2} \cdot 3.5,2^{6}, 3^{4}\right]$} & \\
\hline $\begin{array}{l}(3 n, 4 n, 4 n, 6 n)<12 \\
(1,2,3,12 n)\end{array}$ & {$\left[2.3^{2} .4,2^{6}, 3^{4}\right]$} & \\
\hline$(3,5,5,5)<_{8}(1,2,5,6)$ & {$\left[2.6,2^{4}, 1^{3} .5\right]$} & \\
\hline$(2,2,6,6)<8(1,2,4,6)$ & {$\left[1^{2} .6,2^{4}, 2^{2} .4\right]$} & \\
\hline$(5,5,5,5)<_{9}(1,3,3,5)$ & {$\left[1^{4} .5,3^{3}, 3^{3}\right]$} & \\
\hline$(2,4,4,4)<_{9}(1,3,3,4)$ & {$\left[3^{3}, 3^{3}, 1^{3} \cdot 2.4\right]$} & \\
\hline$(2,7,7,7)<_{10}(1,2,4,7)$ & {$\left[2.4^{2}, 2^{5}, 1^{3} .7\right]$} & \\
\hline$(2,3,6,6)<_{10}(1,2,4,6)$ & {$\left[2.4^{2}, 2^{5}, 1^{2} .2 .6\right]$} & \\
\hline$(8,8,8,8)<12(1,2,4,8)$ & {$\left[1^{4} .8,2^{6}, 4^{3}\right]$} & \\
\hline$(1,3,7,7)<16(1,2,3,7)$ & {$\left[1^{2} .7^{2}, 2^{8}, 1.3^{5}\right]$} & $\Delta(3,7,7) \nless \Delta(2,3,7)$ \\
\hline$(3,5,5,5)<16(1,2,3,10)$ & {$\left[2^{3} \cdot 10,2^{8}, 1.3^{5}\right]$} & \\
\hline$(2,3,4,4)<16(1,2,3,8)$ & {$\left[2^{2} .4 .8,2^{8}, 1.3^{5}\right]$} & \\
\hline$(2,5,5,5)<18(1,2,4,5)$ & {$\left[2.4^{4}, 2^{9}, 1^{3} \cdot 5^{3}\right]$} & \\
\hline$(2,2,8,8)<_{18}(1,2,3,8)$ & {$\left[1^{2} \cdot 4^{2} \cdot 8,2^{9}, 3^{6}\right]$} & \\
\hline$(5,5,5,5)<18(1,2,3,10)$ & {$\left[2^{4} .10,2^{9}, 3^{6}\right]$} & \\
\hline$(2,4,4,4)<18(1,2,3,8)$ & {$\left[2^{3} .4 .8,2^{9}, 3^{6}\right]$} & \\
\hline$(2,4,4,8)<_{21}(1,2,3,8)$ & {$\left[1.2^{2} .8^{2}, 1.2^{10}, 3^{7}\right]$} & \\
\hline$(2,3,8,8)<22(1,2,3,8)$ & {$\left[1^{2} .4 .8^{2}, 2^{11}, 1.3^{7}\right]$} & \\
\hline$(3,4,4,4)<22(1,2,3,8)$ & {$\left[2^{3} .8^{2}, 2^{11}, 1.3^{7}\right]$} & \\
\hline$(3,9,9,9)<24(1,2,3,9)$ & {$\left[1^{3} \cdot 3 \cdot 9^{2}, 2^{12}, 3^{8}\right]$} & \\
\hline
\end{tabular}

\section{References}

1. G. V. Belyŭ: Galois extensions of a maximal cyclotomic field, Izv. Akad. Nauk SSSR Ser. Mat. 43 (1979), no. 2, 267-276, 479.

2. W. Bosma, J. Cannon and C. Playoust: The Magma algebra system. I. The user language, J. Symbolic Comput., 24 (1997), 235-265.

3. A. Breda: A theory of restricted regularity of hypermaps, J. Korean Math. Soc. 43 (2006), No. 5, 991-1018.

4. A. Breda: Riemann surfaces and restrictively-marked hypermaps, Ars Math. Contemp. 3 (2010), 87-98.

5. A. Breda, D. A. Catalano, J. Karabáš and R. Nedela: Maps of Archimedean class and operations on dessins, Discrete Math. 338, Issue 10 (2015), 1814-1825.

6. A. Breda, D. A. Catalano, J. Karabáš and R. Nedela: Quadrangle group inclusions, to appear.

7. A. Breda, D. A. Catalano, J. Karabáš and R. Nedela: Atlas of quadrangle group inclusions, web page, http://www.savbb.sk/ karabas/science.html\#atlas.

8. H. U. Besche, B. Eick, and E. A. O'Brien: The Small Groups library, http://www.icm.tu-bs. de/ag_algebra/software/small/, 2014. 
9. The GAP Group: GAP - Groups, Algorithms, and Programming, Version 4.7.6, 2014, http:// www.gap-system.org.

10. L. Greenberg: Maximal Fuchsian Groups, Bull. Amer. Math. Soc., 69 (1963), 569-573.

11. G. A. Jones and D. Singerman: Complex Functions, an algebraic and geometric viewpoint, Cambridge University Press, Cambridge 1988.

12. S. Katok: Fuchsian groups, The University of Chicago Press, Chicago 1992.

13. S. K. Lando and A. K. Zvonkin: Graphs on Surfaces and Their Applications, Springer 2004.

14. D. Singerman: Subgroups of Fuchsian groups and finite permutation groups, Bull. London Math. Soc., 2 (1970), 319-323.

15. D. Singerman: Finitely maximal Fuchsian groups, J. London Math. Soc. (2), 6 (1972), 29-38.

16. D. Singerman and R. I. Syddall: The Riemann surface of a uniform dessin, Beiträge Algebra Geom. 44 (2003), no. 2, 413-430.

17. J. Wolfart: The 'Obvious' part of Belyi's Theorem and Riemann Surfaces with many automorphisms, pp. 97-112 in Geometric Galois Actions 1, ed. L. Schneps and P. Lochak, London Math. Soc. Lecture Notes Ser. 242, Cambridge University Press, Cambridge, 1997. 


\section{严 Springer}

http://www.springer.com/978-3-319-30449-6

Symmetries in Graphs, Maps, and Polytopes

5th SIGMAP Workshop, West Malvern, UK, July 2014

Širán̆, J.; Jajcay, R. (Eds.)

2016, X, 332 p. 46 illus., Hardcover

ISBN: 978-3-319-30449-6 